\title{
A constant influx model for dike propagation: Implications for magma reservoir dynamics
}

\author{
P. Traversa, ${ }^{1}$ V. Pinel, ${ }^{2}$ and J. R. Grasso ${ }^{1}$ \\ Received 22 April 2009; revised 12 August 2009; accepted 2 September 2009; published 7 January 2010.
}

[1] Most observations of seismicity rate during dike propagation on basaltic volcanoes show (1) rate stationarity despite possible variations of the dike tip velocity, (2) frequent lack of clear and monotonic hypocenter migration following dike propagation, and (3) event occurrences located backward with respect to the dike tip position. On these bases, the origin of the seismicity contemporary to dike intrusion within basaltic volcanoes cannot be solely related to the crack tip propagation. Seismicity rather appears to be the response of the edifice itself to the volumetric deformation induced by the magma intruding the solid matrix. The volume change induced into the volcano edifice over time by the intruding magma is equal to the magma flux injected into the dike from the reservoir. The consequence of this is that the stationary seismicity rate observed during the intrusion is a proxy for the magma flux withdrawn from the reservoir. We consider a two-phase dike propagation model, including a first vertical propagation followed by a lateral migration along a lithological discontinuity. We explore (1) under which geophysical conditions the vertical dike is fed at constant flow rate of magma and (2) dike propagation patterns. Implications entailed by constant volumetric flux on the Piton de la Fournaise volcano case study suggest a minimum size for the magma reservoir of about $1 \mathrm{~km}^{3}$ and a maximum value for the initial magma reservoir overpressure of about 2.2 $\mathrm{MPa}$. Considering similar magma inflow rates during vertical and lateral dike propagation phases, we reproduce independent estimates of propagation velocities, rise times, and injected volumes when applying the model to the August 2003 Piton de la Fournaise eruption.

Citation: Traversa, P., V. Pinel, and J. R. Grasso (2010), A constant influx model for dike propagation: Implications for magma reservoir dynamics, J. Geophys. Res., 115, B01201, doi:10.1029/2009JB006559.

\section{Introduction}

[2] Magma-driven fracture is a commonly observed mechanism that allows to rapidly transport melt through cold and brittle country rock without extensive solidification [Lister and Kerr, 1991]. It therefore differs from porous flow through a deformable and partially molten matrix, which is characteristic of melt generation in the mantle [e.g., McKenzie, 1984] and from slow diapiric rise of granite through viscous country rock [Pitcher, 1979; Rubin, 1993a].

[3] The difficulty of making direct observations of the plumbing system and of the dynamics of conduit formation within volcanoes makes only approximate the knowledge of the parameters and physical balances that govern the propagation of the fissure system.

[4] Previous authors have proposed analytical models of fluid-driven fracture [e.g., Lister, 1990a, 1990b; Lister and Kerr, 1991; Roper and Lister, 2005]. These studies suppose that dikes are fed from a reservoir of magma at depth; the

\footnotetext{
${ }^{1}$ Laboratoire de Géophysique Interne et Tectonophysique, CNRS Université Joseph Fourier, Grenoble, France.

${ }^{2}$ Laboratoire de Géophysique Interne et Tectonophysique, IRD, Université de Savoie, CNRS, Le Bourget du Lac, France.

Copyright 2010 by the American Geophysical Union. 0148-0227/10/2009JB006559\$09.00
}

crack is initiated within the chamber walls, where favorable conditions promote dike propagation, leading to magmatic injections.

[5] The competing pressures, whose balance drives the dike propagation, are (1) the elastic stresses generated by deformation of the host rock; (2) the stresses required to extend the tip against the rock resistance; (3) the buoyancy forces related to the difference between magma and country rock densities; (4) the viscous pressure drop due to magma flow; (5) the magma driving overpressure; and (6) the regional preexisting stress field [e.g., Lister, 1990b; Lister and Kerr, 1991]. In this framework Lister [1990a] concludes that the fracture mechanics only characterize the crack tip zone, while the crack width and the rate of crack propagation are determined by the fluid dynamics. Static or quasi-static solutions for equilibrium crack are therefore inappropriate. It follows that the most important role in the pressure balances is played by pressures $1,3,4$, and 5 . Note that pressure 2 is negligible "soon" away from the crack tip, and pressure 6 mainly acts on the dike orientation [Lister, 1990b; Lister and Kerr, 1991].

[6] In the literature, dike propagation has been modeled according to two basic independent boundary conditions. On the one hand, some authors consider the fluid fracture as driven by a constant overpressure magma chamber at its base [Rubin, 1993b, 1993a; Mériaux and Jaupart, 1998; Roper 
and Lister, 2005]. On the other hand, Lister [1990a, 1990b] assumes a constant influx condition. The first hypothesis has been claimed geologically more appropriate than the second one [e.g., Mériaux and Jaupart, 1998]. The dike growth model from a finite size magma chamber proposed by Ida [1999], however, leads Ida to conclude that only in the case of extremely large and compressible magma reservoirs the melt pressure is actually able to remain constant as the dike propagates.

[7] From the observation point of view, we only have indirect access to dike propagation, the only parameter we can estimate being the propagation velocity, i.e., a few meters per second on basaltic volcanoes. These velocities can be deduced either from observations of the seismic signals associated with the advancing crack tip [Aki et al., 1977; Shaw, 1980; Battaglia et al., 2005], or inferred from the size and composition of xenolithes carried by the flow [Carmichael et al., 1977; Spera, 1980; Pasteris, 1984], or inferred from surface deformation measurements [e.g., Toutain et al., 1992; Battaglia and Aki, 2003; Peltier et al., 2005; Aloisi et al., 2006; Peltier et al., 2007]. As pointed by Battaglia et al. [2005] and Klein et al. [1987], however, well-documented cases of earthquake hypocenters migrating simultaneously to the injected magma toward the surface are rare. A question mark remains on the fact that this lack of well-documented upward an monotonic earthquake migration contemporary to magma ascent prior to an eruption could simply be an artifact due to a poor station coverage on many of the world's active volcanoes [Battaglia et al., 2005]. Available observations suggest however that, while vertical hypocenter migrations are uncommon, horizontal migrations appear to be more frequent (e.g., the 1978 Krafla intrusion [Einarsson and Brandsdottir, 1980] and the 2000 Izu Islands magma migration [e.g., Toda et al., 2002]).

[8] From scale-invariance explorations [Grasso and Bachèlery, 1995] and theoretical considerations [Rubin and Gillard, 1998], the distribution of recorded dike-induced earthquakes is suggested to map the distribution of rock mass sites that are near to failure, and does not necessarily reflect the extent of the dike. To note that only in the case of an homogeneous medium the maximum deformation occurs at the dike head, where we therefore expect most of the seismicity to occur [Lister, 1990a; Pinel and Jaupart, 2004]. Besides, earthquakes generated from the tensile propagation of the dike tip are likely to be too small in magnitude [Rubin, 1995; Rubin et al., 1998] and too high in frequency [Cornet, 1992] to be detected by standard seismic networks that operate at volcano surface. The shear type of the generally recorded seismicity accompanying magma movement, moreover, is not compatible with the signal associated to a dynamic propagation of the dike tip (i.e., a tensile fracture) [Cornet, 1992].

[9] Observations of volcano-tectonic (VT) seismicity during dike propagation on basaltic volcanoes show a constant seismicity rate over time [Traversa and Grasso, 2009]. This characteristic pattern for the seismic signature of dike propagation demonstrates to be reproducible on different volcanoes: Piton de la Fournaise (PDLF), seven dike intrusions in the period 1988-1992; Etna, 2002 dike intrusion; and Miyakejima (MI), 2000 dike intrusion.

[10] For the Piton de la Fournaise dike intrusions, Traversa and Grasso [2009] report diffuse VT seismicity within the shallow edifice. On this basis, Traversa and Grasso [2009] argue for the seismicity generated during dike injection to be a generic response of the volcanic edifice to the intrusion instead of an accurate mapping of the dike tip propagation.

[11] Toda et al. [2002] show that the change in seismicity rate generated by the 2000 dike intrusion at Izu Islands (Japan) scales with the change in stressing rate induced by the propagation and opening of the dike. This result demonstrates that the stressing rate governs the seismicity. It moreover supports the hypothesis of magma flow rate scaling with the seismicity rate [Pedersen et al., 2007].

[12] All these argue for the stationary seismicity rate accompanying the dike propagation to be the response of the brittle lithosphere to a constant volumetric deformation rate (i.e., a constant influx of magma over time) induced by the intrusion [e.g., Traversa and Grasso, 2009].

[13] Following Traversa and Grasso's [2009] observations, the aim of this paper is therefore primarily (1) to analyze how a constant flow rate of magma injected into the dike from the reservoir is consistent with the dynamics of a fluid-driven fracture propagating under realistic conditions for the magma chamber overpressure and (2) to evaluate the implications for the volcano dynamics. This is achieved by considering a two-phase dike propagation model involving an initial vertical propagation phase followed by a horizontal migration phase.

[14] Such two-phase propagation style for dike propagating from a magma source at shallow depth to the surface, is commonly observed on basaltic volcanoes worldwide, e.g., Mt. Etna (southern Italy) [e.g., Aloisi et al., 2006]; Miyakejima (southern Japan) [e.g., Nishimura et al., 2001]; and in particular on Piton de la Fournaise [e.g., Toutain et al., 1992; Bachèlery, 1999; Peltier et al., 2005, 2007].

[15] For the vertical rise of a buoyant fluid-filled crack from a shallow storage system toward the surface, we consider two boundary conditions at the dike inlet, constant and variable reservoir overpressure. In the latter case the overpressure variation is controlled by the withdrawal of magma from the chamber induced by the dike growth. Subsequently, the effect of a lithological discontinuity at depth is introduced by reducing the buoyancy of the fluid in the upper layer. This density step induces a slow down of the rising magma and favors melt accumulation and subsequent lateral dike propagation.

[16] We apply the two-phase dike propagation model to the magmatic intrusion that fed the August 2003 Piton de la Fournaise (PDLF) eruption. The stationary rate of VT earthquakes accompanying the August 2003 PDLF dike intrusion supports the result found by Traversa and Grasso [2009] in the $1992-1996$ period. Accordingly we expect stationary flux of magma to feed the propagating dike. Besides, the number of works devoted to its study make it one of the best studied intrusive episodes observed on PDLF volcano in the last years.

[17] This application allows us to derive possible generic implications on the mechanisms driving magma movements on basaltic volcanoes. This so-called "proximal" eruption (according to Peltier et al.'s [2008] classification) is a good example to use to validate our model, first because it is accompanied by a stationary seismicity rate over time, and second because it is constituted of a vertical and lateral phase dike propagation, which is the generally accepted feature 

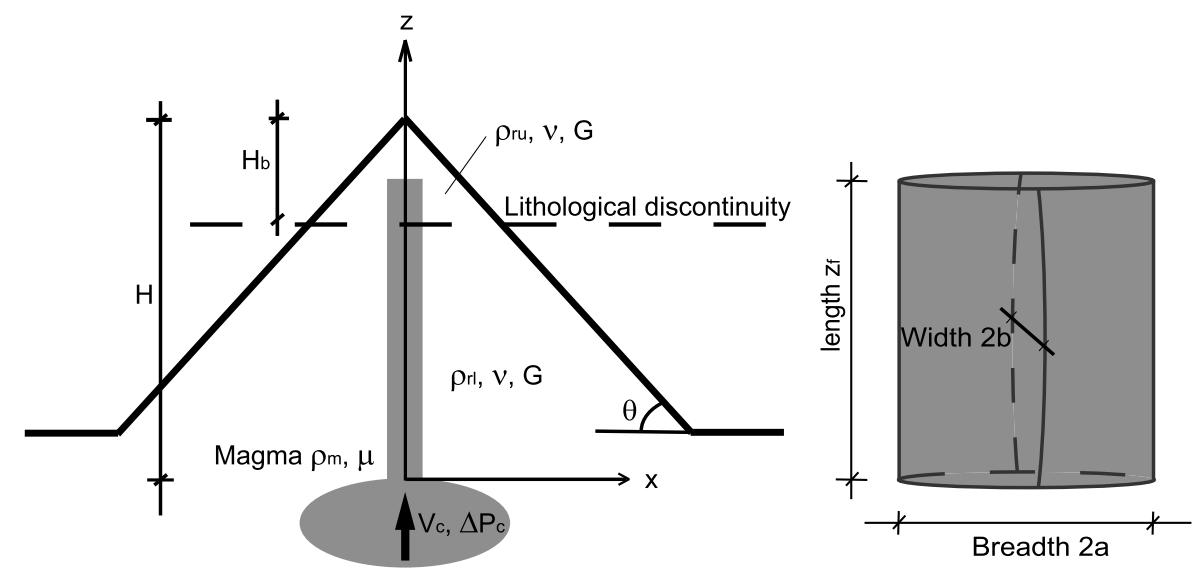

Figure 1. (left) Sketch illustrating the geometry of a vertical dike and (right) the shape of the fissure; $2 b \ll$ $2 a \leq z_{f}$. Half breadth $a$ is assumed a priori.

describing flank eruptions at PDLF volcano [e.g., Toutain et al., 1992; Bachèlery et al., 1998; Bachèlery, 1999; Peltier et al., 2005, 2007].

\section{Models of Dike Propagation}

\subsection{Vertical Dike Propagation}

[18] In this section we focus on the vertical propagation of a buoyant fluid-filled crack, from a shallow storage system toward the surface (see Figure 1). The crack is fed from a magma reservoir whose overpressure $\Delta P_{c}$ is either constant over time, or evolves as a consequence of the withdrawal of magma from the reservoir. In particular, the aim of this section, is to individuate whether and under which conditions, a magma reservoir is able to feed a propagating dike with constant flux of magma input from the reservoir.

\subsubsection{Model Description}

[19] For simplicity we consider a two-layer elastic halfspace, characterized by Poisson ratio $\nu$ and shear modulus $G$ and subject to a lithostatic stress field. The magma-filled fracture originates from the roof of a magma reservoir located at depth $H$, which is taken as the reference level. The $z$ axis is oriented positively upward, with $z=0$ at the reference level, where magma (of density $\rho_{m}$ ) has developed the overpressure $\Delta P_{c}$ with respect to the surroundings. A lithological discontinuity is located at depth $H_{b}$, such that the rock density as a function of depth is given by (see Figure 1)

$$
\begin{array}{ll}
\text { Lower layer } & \rho_{r}(z)=\rho_{r l} \text { for } z<H-H_{b} \\
\text { Upper layer } & \rho_{r}(z)=\rho_{r u} \text { for } z>H-H_{b} .
\end{array}
$$

As demonstrated by previous authors [e.g., Lister, 1990a, 1990b; Lister and Kerr, 1991], once the dike length is large enough, the influence of the toughness of rocks on dike propagation can be neglected. The fluid-filled crack propagation is in fact dominated by fluid dynamics, except during the early nucleation of the crack [Lister, 1990a]. On these bases, we neglect the strength of the surrounding rocks in the force balance, and hence do not treat stress singularity at the tip. We focus instead on the interplay between buoyancy, viscous head loss and elastic stresses. By considering also flow-induced stresses, the stress induced by the dike opening is given by [Pinel and Jaupart, 2000]

$$
\sigma_{o}(z)=\Delta P_{c}+\sigma_{b}(z)+p_{v},
$$

where $p_{v}$ is the viscous head loss and $\sigma_{b}(z)$ is the magma overpressure due to buoyancy. $\sigma_{b}(z)$ is given by

$$
\sigma_{b}(z)=\int_{0}^{z}\left(\rho_{r}\left(z^{\prime}\right)-\rho_{m}\right) g d z^{\prime}
$$

Following Pinel and Jaupart [2000] and Maaløe [1998], we fix the dike breadth $a$, and we assume that the dike adopts an elliptical cross section with semiaxes $a$ and $b$ characterized by $b(z, t) \ll a$ (see Figure 1$)$. In this case, the dike-induced stress is given by [Muskhelishvili, 1963]

$$
\sigma_{o}(z, t) \approx \frac{G}{1-\nu} \frac{b(z, t)}{a}
$$

Magma is considered as Newtonian, viscous and incompressible. Flow proceeds in a laminar regime. According to Pinel and Jaupart [2000], we obtain the following equation for the case of null lateral stress variation:

$$
\frac{\partial b(z, t)}{\partial t}=-\frac{1}{4 \mu} \frac{\partial}{\partial z}\left(\frac{\partial \sigma_{b}}{\partial z} b^{3}\right)+\frac{G}{16 \mu a(1-\nu)} \frac{\partial^{2} b^{4}}{\partial z^{2}},
$$

where $\mu$ is magma viscosity.

[20] We scale the pressures by the initial overpressure within the magma reservoir, $\Delta P_{c}(t=0)=\Delta P_{0}$, and the front height $z_{f}$ by the reservoir depth $H$. Scales for time, flux and fracture width for the vertical propagation are

$$
\begin{gathered}
{[t]=\frac{16 \mu H^{2} G^{2}}{\Delta P_{0}^{3} a^{2}(1-\nu)^{2}},} \\
{[Q]=\frac{(1-\nu)^{3} \Delta P_{0}^{4} a^{4}}{16 G^{3} \mu H},} \\
{[b]=\frac{\Delta P_{0} a(1-\nu)}{G} .}
\end{gathered}
$$


These are the reference quantities in the computation, i.e., $[t]$ is the timescale for opening the crack over a length $H$ with a uniform overpressure $\Delta P_{0}$. Length scale $[b]$ is the fracture width originated by an overpressure $\Delta P_{0}$. The scale for the dike propagation velocity is then given by: $[v]=H /[t]$. The initiation of the fracture on the reservoir walls is imposed a priori with an elliptical profile. This affects the fracture growth only for a duration needed for an initial adjustment stage $[I d a, 1999]$. We can define three dimensionless numbers. The dimensionless number $R_{1 l}$ characterizes the magnitude of the buoyancy force scaled to the initial overpressure, as follows:

$$
R_{1 l}=\frac{\left(\rho_{m}-\rho_{r l}\right) g H}{\Delta P_{0}}
$$

Dimensionless numbers $R_{1 u}$ and $R_{2}$ characterize the lithological discontinuity:

$$
\begin{gathered}
R_{1 u}=\frac{\left(\rho_{m}-\rho_{r u}\right) g H}{\Delta P_{0}} \\
R_{2}=\frac{H_{b}}{H} .
\end{gathered}
$$

We therefore have the following dimensionless problem to solve:

$$
\begin{gathered}
\frac{\partial b(z, t)}{\partial t}=-4 \frac{\partial}{\partial z}\left(\frac{\partial \sigma_{b}}{\partial z} b^{3}\right)+\frac{\partial^{2} b^{4}}{\partial z^{2}} \\
b_{(z=0, t)}=\Delta P_{c}(t) .
\end{gathered}
$$

When there is no lithological discontinuity, $R_{1 l}=R_{1 u}=R_{1}$, and equation (12) reduces to

$$
\frac{\partial b(z, t)}{\partial t}=4 R_{1} \frac{\partial b^{3}}{\partial z}+\frac{\partial^{2} b^{4}}{\partial z^{2}}
$$

This is solved numerically using a semi-implicit finite difference scheme with Dirichlet boundary conditions.

[21] In this framework, equation (12) allows to follow the dynamics of dike propagation on its way toward the surface. We checked that mass conservation was satisfied on the scale of the whole dike, which requires the instantaneous volume change to be equal to the basal flux, both values being issued from the numerical computation. The dimensions of the fracture at its base (i.e., the imposed $a$ value and the calculated $b(0, t)$, which depends on the overpressure at the dike inlet) determine the volume of magma intruding into the fissure per time unit. The velocity of the dike propagating toward the surface is given by $d z_{f} / d t$, where $z_{f}$ is the fracture front height (see Figure 1).

[22] When magma is injected from the reservoir into the dike, it induces a decrease of the magma reservoir volume $\Delta V_{c}$, which might in turn induce a decrease of the reservoir overpressure $\Delta P_{c}$ as well. Considering the elastic deformation induced by a point source (i.e., the magma reservoir) embedded in an infinite medium, the evolution of the reservoir overpressure follows the equation (V. Pinel and C. Jaupart, personal communication, 2009)

$$
d \Delta P_{c}(t)=\frac{d V_{c}(t)}{V_{c}(t)} \frac{4 K G}{4 G+3 K}
$$

where $K$ is the magma bulk modulus. The volume variation in the magma reservoir can be related to the volume of magma injected into the dike by

$$
d V_{c}(t)=-Q(t) d t
$$

with $Q$ the flux of magma entering the dike. When magma is fully compressible, $K=0$ and the magma reservoir overpressure remains constant trough time. For incompressible magma, $K \rightarrow \infty$ and equation (15) becomes

$$
d \Delta P_{c}(t)=\frac{d V_{c}(t)}{V_{c}(t)} \frac{4 G}{3}
$$

To fully describe the evolution of the reservoir pressure, we introduce two new dimensionless numbers:

$$
R_{3}=\frac{\Delta P_{0} a^{2}(1-\nu) H}{G V_{c}},
$$

which is the inverse dimensionless reservoir volume, and

$$
R_{4}=\frac{4 K G}{\Delta P_{0}(4 G+3 K)}
$$

which relates the overpressure variation in the reservoir to the initial overpressure value.

\subsubsection{Results}

[23] We study the propagation of a vertical dike from a shallow reservoir, according to the geometry illustrated in Figure 1. We investigate under which conditions the magma flux injected into the dike remains constant during dike growth. Using the dimensionless numbers above described, we discuss the role played by each parameter in determining the regime of magma flux carried by the rising dike. We solve the problem for three different configurations: (1) dike rising from a constant overpressure magma reservoir in a homogeneous medium, (2) dike rising from a variable overpressure magma reservoir in a homogeneous medium, and (3) dike rising from a variable overpressure magma reservoir in a layered medium.

[24] First, we consider the case of a dike rising from a constant overpressure magma reservoir $\left(\Delta P_{c}=\Delta P_{0}=\right.$ const $)$ in a homogeneous medium (i.e., $\rho_{r l}=\rho_{r u}, R_{1 l}=R_{1 u}=R_{1}$ ). As shown in Figure 2, after some numeric adjustment iterations (whose number decreases with $R_{1}$ value), the flux of magma in the growing dike evolves similarly to the propagation velocity (Figures $2 \mathrm{a}$ and $2 \mathrm{~b}$ ). This is related to the fact that, in this case, the dike growth depends on tip propagation. Since fracture half breadth $a$ is assumed constant a priori and the medium is homogeneous, the dike only grows along the propagation direction (Figure $2 \mathrm{c}$ ). In this first case, the only dimensionless number affecting the regime of magma flux over time is $R_{1}$. We consider as negligible a flux variation less 

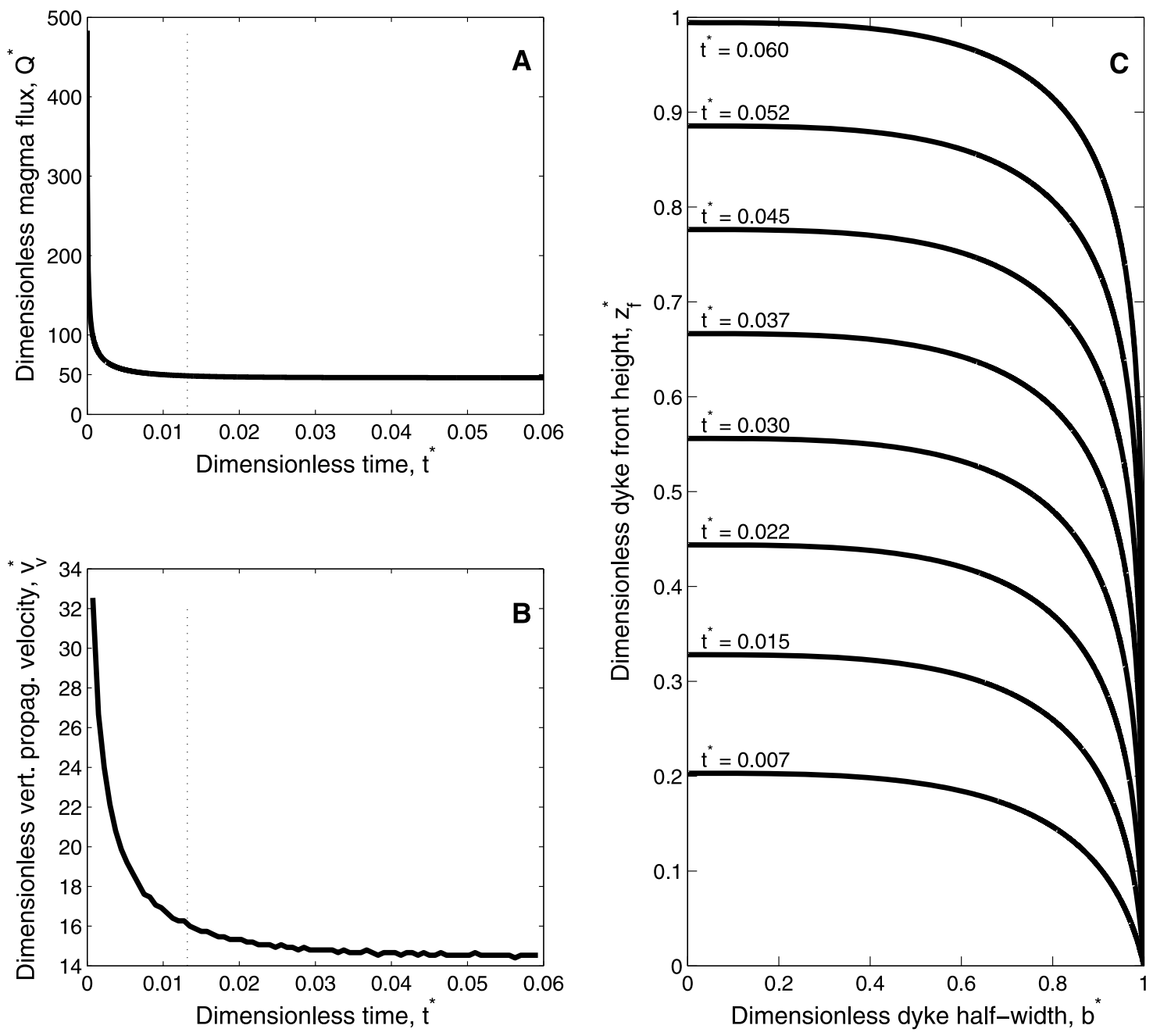

Figure 2. Magma-filled dike rising in a homogeneous medium from a constant overpressure magma chamber at depth. (a) Dimensionless magma flux injected into the dike over time; (b) dimensionless propagation velocity versus time; (c) evolution of the crack shape for progressive growth stages. $R_{1}\left(R_{1}=\right.$ $\left.\left(\rho_{m}-\rho_{r}\right) g H / \Delta P_{0}\right)$ value used in the calculation is -3.55 . Dotted lines in Figures 2a and 2b indicate $z_{f}^{*}=0.3$. Here $t=t^{*}[t], Q=Q^{*}[Q], v_{v}=v_{v}^{*}[v], b=b^{*}[b], z_{f}=z_{f}^{*}[H]$, where scales for time [t], flux [Q], and fracture width $[b]$ are given in equations (6) to (8); lengths are scaled by the reservoir depth $H$; and scale for propagation velocity is $[v]=[H] /[t]$.

than $5 \%$ between dimensionless dike heights $z_{f}=0.3$ and $z_{f}=$ 0.9. The choice of the first limit is imposed by discarding initial numerical adjustment iterations. As shown in Figure 3 (black open squares), the magma flux withdrawn from the reservoir remains constant during dike rising for $R_{1} \leq-3.55$. In this constant overpressure case, and for a given reservoir depth, the only parameter determining the regime of the magma flux carried by the growing dike is the ratio between the buoyancy force and the magma overpressure at the dike inlet.

[25] Second we consider the same case as above, but with the reservoir overpressure varying as magma is withdrawn. Through the dimensionless numbers $R_{3}$ and $R_{4}$, we explore the role of the magma chamber volume $V_{c}$ and of the magma bulk modulus $K$, which relates changes in reservoir volume with changes in pressure, on the regime of magma flux withdrawn from the reservoir. As illustrated in Figure 3 (solid symbols), the smaller the dimensionless number $R_{3}$, the more the flux tends to remain constant during dike propagation and vice versa. It means that the larger the chamber volume with respect to the dike scale volume, the more negligible a withdrawal of magma is in terms of variations in magma flow rate and reservoir overpressure during dike rising. In the same way, the smaller the dimensionless number $R_{4}$, the smaller the magma flux variation obtained during dike rising and vice versa. This implies that the more the magma tends to be incompressible, i.e., $K \rightarrow \infty$, the more the flow of magma injected into the dike varies over time as the dike propagates. As shown in Figure 3 legend, this scenario corresponds to larger variations in the reservoir overpressure $\left(\Delta P_{c}\right.$ variation) face to the withdrawal of magma from the reservoir. Conversely, more compressible magmas, i.e., $K \rightarrow 0$, allow for smaller variations in the magma flow rate over time, which correspond to smaller overpressure variations accompanying magma withdrawn from the reservoir. However, only small overpressure variations $\left(\Delta P_{c}\right.$ variation less than $\sim 2 \%$ ) in the magma reservoir allow for the magma flow rate to remain constant during dike propagation. 


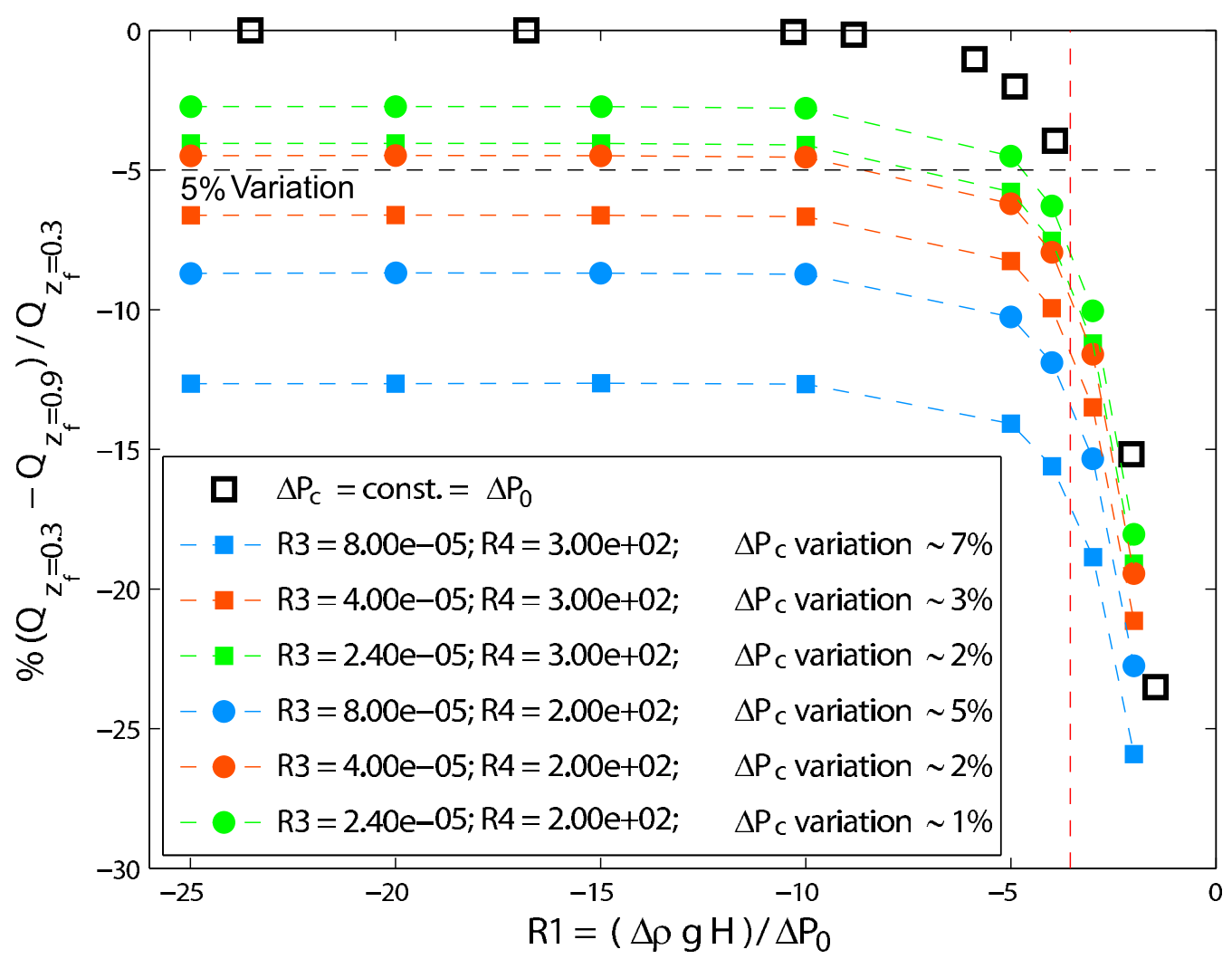

Figure 3. Percentage of magma influx variation during dike growth within a homogeneous medium as function of the dimensionless number $R_{1}\left(R_{1}=\left(\rho_{m}-\rho_{r}\right) g H / \Delta P_{0}\right)$. Black squares indicate constant overpressure at the dike inlet; colored symbols indicate variable overpressure in the chamber. Color of solid symbols is related to the $V_{c}$ value; circles or squares depend on the $K$ value. Reservoir overpressure variations $\Delta P_{c}$ variation indicated in the legend are issued from the computation.

[26] As a third case we consider a lithological discontinuity within the volcanic edifice. This discontinuity is intended in terms of rock densities, which are chosen such that magma has intermediate density between the lower and upper rock layers $\left(\rho_{r l}<\rho_{m}<\rho_{r u}\right)$. This allows for considering a twofold effect: on one hand the higher fracture density of the solid medium close to the surface, which implies a lower density of the shallow layer and, on the other hand, the fact that magma degasses while rising, becoming more and more dense as approaching the surface. The effect of this density step is to slow down the rise of magma, creating favorable conditions for magma accumulation at the discontinuity depth $H_{b}$.

[27] Figure 4 illustrates the variation of the dimensionless magma flux, propagation velocity, and dike shape during dike propagation from an overpressured magma chamber, in a two-layer medium. After an initial numeric adjustment transient, the magma flux remains constant over time, being blind to the lithological discontinuity (Figure 4a). The dike volume continues therefore to regularly grow as dike rises. On the other hand, the dike propagation velocity, computed as $d z_{f} / d t$, significantly decreases when the dike reaches the depth of the density step (Figure 4b), as also shown by Taisne and Jaupart [2009].

[28] Reminding that the seismic response of a volcanic edifice to dike propagation is reported to be stationary over time [Traversa and Grasso, 2009], this result supports the hypothesis of scaling between seismicity rate accompanying the dike intrusion and the volumetric flux of magma entering the dike. On the other hand, it excludes the possibility of a direct scaling between the seismicity rate and the dike propagation velocity. The density step does not affect the shape of the fracture at the dike inlet (Figure 4c). In our model, for a given magma viscosity, the magma flux supply only depends on the shape of the crack at the junction with the reservoir roof. It can therefore remain constant over time as dike grows.

[29] While dike half breadth $a$ is assumed to be constant over time, the dimensionless numbers $R_{1 r l}, R_{1 r u}$ and $R_{2}$ play a role in determining the width of the dike at the inlet, and therefore the regime of magma flux carried by the propagating dike. The parameter $R_{1 r l}$ has been discussed above, while Figure 5 shows the effect of $R_{1 r u}$ and $R_{2}$ dimensionless numbers on the regime of magma flow over time. In analogy with the previous discussion, we consider as negligible a variation in the magma flux less than $5 \%$ between dimensionless front heights $z_{f}=0.3$ and 0.9 . Variation in magma flux during dike rise are negligible for $R_{1 r u}<1.5$ and for $R_{2}<$ 0.5 . These imply that, in order for the flux of magma to remain constant over time, the densities of the magma and the upper layer should be quite close in value, and that the discontinuity should not be deeper than half the reservoir depth.

[30] As shown in Figure 13c, when magma buoyancy faints, due to a decrease in the surrounding rock density, an inflation starts to grow at the dike head. Here elastic stresses may exceed the rock toughness and new fractures may initiate. 

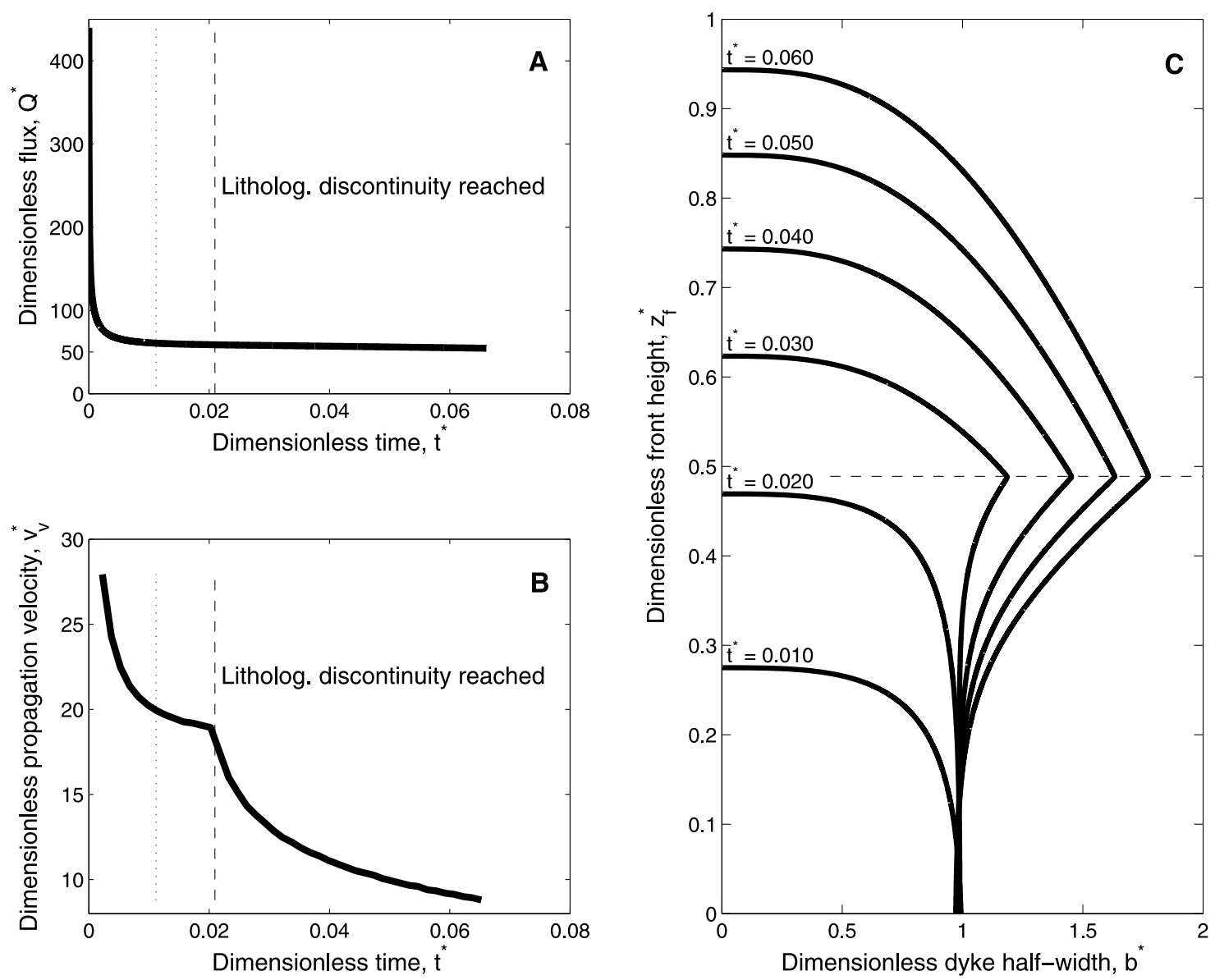

Figure 4. Magma-filled dike rising in a homogeneous medium from a constant overpressure magma chamber at depth. (a) Dimensionless magma flux injected into the dike over time; (b) dimensionless propagation velocity versus time; (c) evolution of the crack shape for progressive growth stages. Parameter values used in the computation are $R_{1 l}=-4.82, R_{1 u}=1.37, R_{2}=0.51, R_{3}=6.9 \times 10^{-9}, R_{4}=1.125$. Dotted lines in Figures $4 \mathrm{a}$ and $4 \mathrm{~b}$ indicate $z_{f}^{*}=0.3$. Here $t=t^{*}[t], Q=Q^{*}[Q], v_{v}=v_{v}^{*}[v], b=b^{*}[b], z_{f}=z_{f}^{*}[H]$, where scales for time $[t]$, flux $[Q]$ and fracture width $[b]$ are given in equations (6) to (8), lengths are scaled by the reservoir depth $H$, and scale for propagation velocity is $[v]=[H] /[t] ; R_{1 u}=\left(\rho_{m}-\rho_{\text {ru }}\right) g H / \Delta P_{0}, R_{1 l}=$ $\left(\rho_{m}-\rho_{r l}\right) g H / \Delta P_{0}, R_{2}=H_{b} / H, R_{3}=\left(\Delta P_{0} a^{2}(1-\nu) H\right)\left(G V_{c}\right), R_{4}=4 K G /\left(\Delta P_{0}(4 G+3 K)\right)$.

\subsection{Lateral Propagation at the Level of Neutral Buoyancy}

[31] Exhaustive description of the solution for dike propagation at a lithological boundary fed by either, constant flux or constant volume of magma is given by Lister [1990b] and Lister and Kerr [1991]. They assume that buoyancy forces do not depend on horizontal distance. The effects of lateral variations of the stress field induced by a volcanic edifice load on the lateral propagation are studied by Pinel and Jaupart [2004]. In this paper we consider an horizontal lithological boundary located within the volcanic edifice. We therefore adapt the solutions given by Pinel and Jaupart [2004] in order to take into account the variation of the external lithostatic pressure induced by the volcano slope along the propagation direction.

\subsubsection{Model Description}

[32] Figure 6 illustrates the geometry and main parameters used in this section. The parameters $\rho_{r u}$ and $\rho_{r l}$ are the rock densities in the upper and lower layer, respectively. For this case, we define the origin of the vertical coordinate $z$ at the discontinuity level, oriented positive upward. The vertical extension of the dike is called $2 a(x)$. The parameters $z_{u}(x)$ and $z_{l}(x)$ stand for the positions of the upper and lower dike tips, respectively, such that we have

$$
2 a(x)=z_{u}(x)-z_{l}(x)
$$

We also define

$$
m=\frac{z_{u}+z_{l}}{z_{u}-z_{l}} .
$$

We neglect the effects of the free surface [Pinel and Jaupart, 2004], so that the stress generated by the pressure difference between the interior and the exterior of the dike, $\sigma_{o}$, is given by

$$
\begin{gathered}
\sigma_{o}(x, z)=\left(\rho_{r u}-\rho_{m}\right) g z-\sigma_{l}(x)+p, \quad \text { if } z>0 \\
\sigma_{o}(x, z)=\left(\rho_{r l}-\rho_{m}\right) g z-\sigma_{l}(x)+p, \quad \text { if } z<0,
\end{gathered}
$$



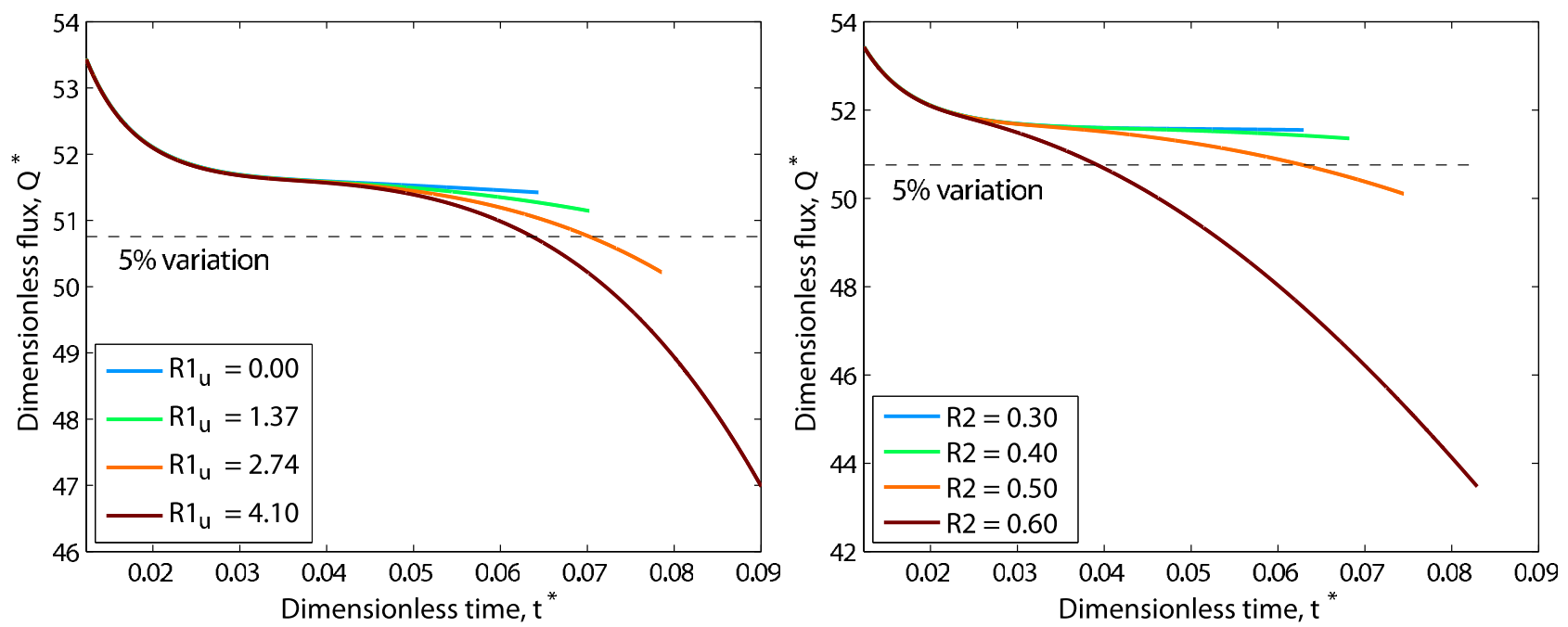

Figure 5. (left) Effect of the dimensionless number $R_{1 r u}$ on the magma flux evolution over time during dike propagation, $R_{2}=0.43$. (right) Effect of the dimensionless number $R_{2}$ on the magma flux evolution over time during dike propagation, $R_{1 r u}=1.37$. For both cases, $R_{1 r l}=-4.1, V_{c}=5 \mathrm{~km}^{3}$ and $K=1 \times 10^{9} \mathrm{~Pa}$. Final time corresponds to surface attainment. $R_{1 u}=\left(\rho_{m}-\rho_{r u}\right) g H / \Delta P_{0}, R_{1 l}=\left(\rho_{m}-\rho_{r l}\right) g H / \Delta P_{0}, R_{2}=H_{b} / H$, $R_{3}=\left(\Delta P_{0} a^{2}(1-\nu) H\right)\left(G V_{c}\right), R_{4}=4 K G /\left(\Delta P_{0}(4 G+3 K)\right)$.

where $p$ is the internal magma pressure, which varies due to viscous friction, and $\sigma_{l}$ is the lithostatic pressure at the lithological boundary, defined by

$$
\sigma_{o}(x)=\rho_{r u} g\left(H_{b}-\theta x\right)
$$

with $\theta$ the volcano slope.

[33] We consider that the lateral dike length is larger than its height and we neglect vertical pressure gradients due to upward flow within the dike [Lister and Kerr, 1991; Pinel and Jaupart, 2004]. In this case, the internal magma pressure $p$ depends only on the lateral position $x$. As before, the condition for the crack to remain open is $\sigma_{o}>0$.

[34] We consider that the dike propagates in damaged rocks, and therefore we set to zero the stress intensity factor at both dike tips [Mériaux et al., 1999]. Following Pinel and Jaupart [2004], this leads to

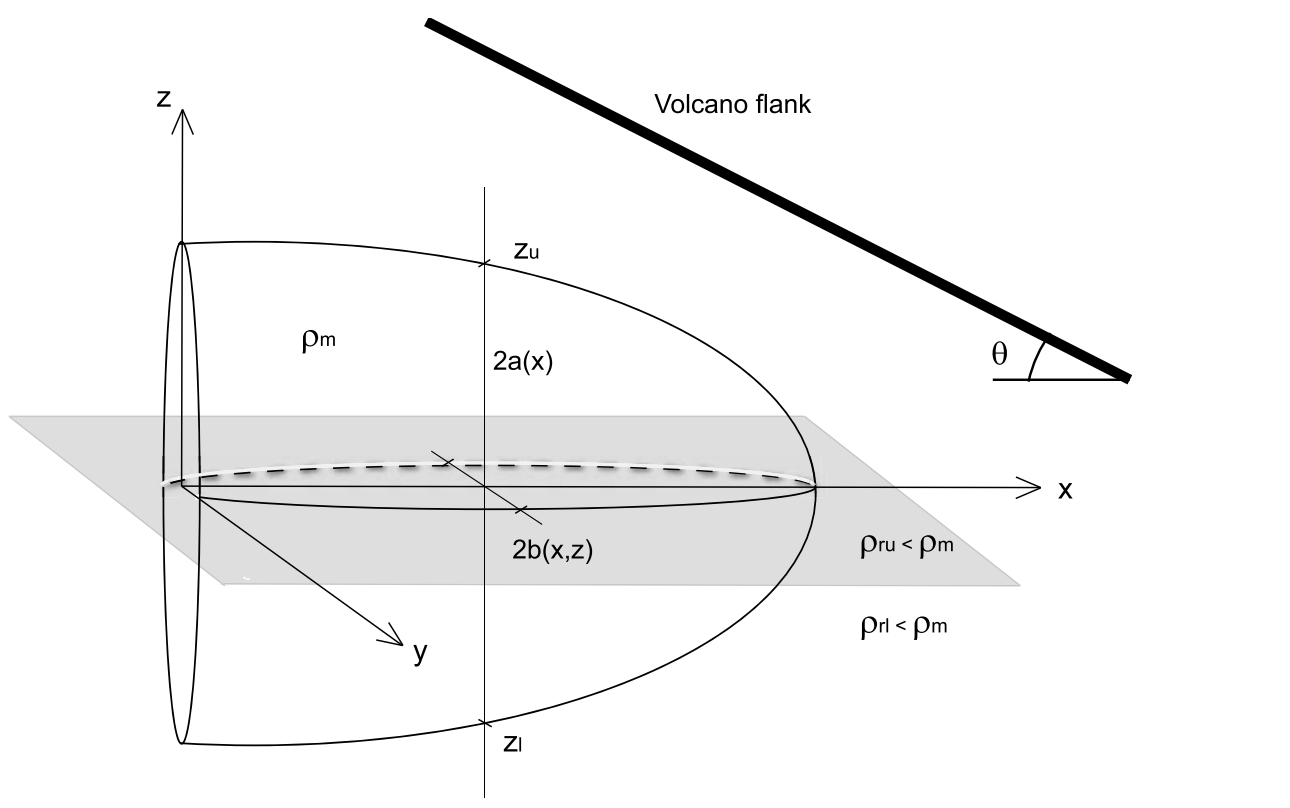

Figure 6. Sketch illustrating the geometry and the main parameters of a dike horizontally propagating at the level of neutral buoyancy. 
It means that for given values of densities $\rho_{r u}, \rho_{r l}$ and $\rho_{m}$, once the overpressure at the lithological discontinuity is known at a given lateral distance $x$, there is a unique solution for the half height $a(x)$ and the tip locations $z_{u}(x)$ and $z_{l}(x)$. This solution can be subsequently used to calculate the dike width $b(x, z)$ using the solution derived from Pinel and Jaupart [2004]. For $-1<s<1$, the half width $b(s)$ is given by

$$
\begin{aligned}
b(s, x)= & \frac{(1-\nu) \sigma_{o}(x, z=0)}{G} \sqrt{1-s^{2}} \\
& +\frac{a(x)(1-\nu) g\left(\rho_{r l}-\rho_{r u}\right)}{G \pi} \\
& \cdot\left[\sqrt{1-s^{2}}\left(-\frac{1}{2} \sqrt{1-m^{2}}-\frac{1}{2} s \arcsin m-m \arcsin m\right)\right. \\
& -\frac{1}{2}(s+m)^{2} \ln \left|\frac{1+s m+\sqrt{\left(1-s^{2}\right)\left(1-m^{2}\right)} \mid}{s+m}\right| \\
& \left.+\frac{\rho_{r u}+\rho_{r l}-2 \rho_{m}}{\rho_{r l}-\rho_{r u}} \sqrt{1-s^{2}}\left(\frac{1}{4} s \pi+\frac{1}{2} m \pi\right)\right],
\end{aligned}
$$

where $s$ is defined by

$$
s=\frac{z}{a(x)}-m .
$$

From equation (25), we can see that dike extension in the upper medium is equal the extension in the lower medium $(m=0)$ just in case $\rho_{r l}-\rho_{m}=\rho_{m}-\rho_{r u}$. As there is no lateral variations of the stress field vertical gradient, $m$ is a constant.

[35] The dike internal pressure $\sigma_{o}$, which keeps the dike open, varies laterally because of both, the volcano flank slope and the viscous head losses due to horizontal magma flow. Magma is considered as Newtonian, viscous and incompressible. Flow proceeds in laminar regime.

[36] Following Pinel and Jaupart [2004] analytical procedure, the dike half height $a(x, t)$, is the solution of

$$
\begin{aligned}
& c_{1} g\left(\rho_{r u}-\rho_{m}\right) \frac{\partial a(x, t)^{3}}{\partial t}=\frac{c_{3}(1-\nu)^{2}}{3 \mu G^{2}} \frac{\partial}{\partial x} \\
& \cdot\left[a(x, t)^{7} g^{3}\left(\rho_{r u}-\rho_{m}\right)^{3}\left(\frac{g\left(\rho_{r l}-\rho_{r u}\right)}{\pi}(1-m)^{3 / 2} \frac{\partial a(x, t)}{\partial x}-\rho_{r u} g \theta\right)\right] .
\end{aligned}
$$

where

$$
\begin{gathered}
c_{n}=\int_{-1}^{1} f(s)^{n} d s \\
f(s)=\frac{G b(s)}{g(1-\nu)\left(\rho_{r u}-\rho_{m}\right) a(x)} .
\end{gathered}
$$

We scale the pressures by the lithostatic load of the rock mass above the density step,

$$
[P]=\rho_{r u} g H_{b},
$$

the flux by the input flux of magma $Q_{i n}$, and all length dimensions by the depth of the lithostatic discontinuity $H_{b}$.
The scale for the time refers to the opening of a fissure over a length $H_{b}$ with a magma flux equal to $Q_{i n}$ and is given by

$$
[t]=\left(\frac{\mu(1-\nu) H_{b}^{9}}{G Q_{i n}^{3}}\right)^{1 / 4}
$$

As shown by Pinel and Jaupart [2004], two dimensionless numbers can be defined:

$$
\begin{gathered}
N_{1}=\frac{3 Q_{i n}^{3 / 4} \mu^{3 / 4} G^{9 / 4}}{H_{b}^{9 / 4}(1-\nu)^{9 / 4}[P]^{3}} \\
N_{2}=-\frac{2 H_{b}^{3}(1-\nu)^{3}[P]^{4}}{3 \mu Q_{i n} G^{3}} .
\end{gathered}
$$

Equation (29) can be rewritten in the dimensionless form

$$
\begin{aligned}
\frac{c_{1}}{c_{3}} N_{1} \frac{\rho_{r u}-\rho_{m}}{\rho_{r u}} \frac{\partial a^{3}}{\partial t}= & -\theta \frac{\left(\rho_{r u}-\rho_{m}\right)^{3}}{\rho_{r u}^{3}} \frac{\partial a^{7}}{\partial x} \\
& +\frac{(1-m)^{3 / 2}\left(\rho_{r u}-\rho_{m}\right)^{3}\left(\rho_{r l}-\rho_{r u}\right)}{8 \pi \rho_{r u}^{4}} \frac{\partial^{2} a^{8}}{\partial x^{2}} .
\end{aligned}
$$

The dimensionless flux is given by

$\frac{q}{Q_{i n}}=N_{2} c_{3} a(x, t)^{7} \frac{(1-m)^{3 / 2}\left(\rho_{r u}-\rho_{m}\right)^{3}\left(\rho_{r l}-\rho_{r u}\right)}{8 \pi \rho_{r u}^{4}}\left[\frac{\partial a(x, t)}{\partial x}-\theta\right]$.

We solve numerically this equation with a semi-implicit finite difference scheme with a Neumann boundary conditions at the source $(x=0)$.

\subsubsection{Results}

[37] In this section we discuss the effect of the model parameters on the propagation of a dike at a lithological boundary, fed by a constant flux of magma. As discussed in the previous section, the dike propagation is affected by the variation in the external lithostatic pressure induced by the volcanic slope along the propagation direction, while vertical stress gradients do not vary laterally.

[38] Lister [1990b] discusses the case of a dike fed by constant flux or constant volume of magma, laterally propagating in a medium with no lateral stress variations. In this case the breadth of the dike $(2 a(x)$ in Figure 6$)$ varies in time all along its length, being however always largest at the origin $(2 a(x=0))$. Pinel and Jaupart [2004] consider the effect of the volcanic edifice load on the propagation of a lateral dike at depth. In this case, the breadth of the dike varies at the head during lateral propagation, due to lateral variations of vertical stress gradients. For the present case, the lateral stress variations are only due to the flank slope of the edifice. Figure 7 shows that, with small flank slopes $(\theta \rightarrow 0)$, the breadth of the dike grows at the origin as the dike propagates, reminding the case discussed by Lister [1990b]. With higher flank slopes, the half breadth $a$ tends to a constant value as the dike laterally propagates. Such constant value does not depend on the propagation distance from the origin. In this 

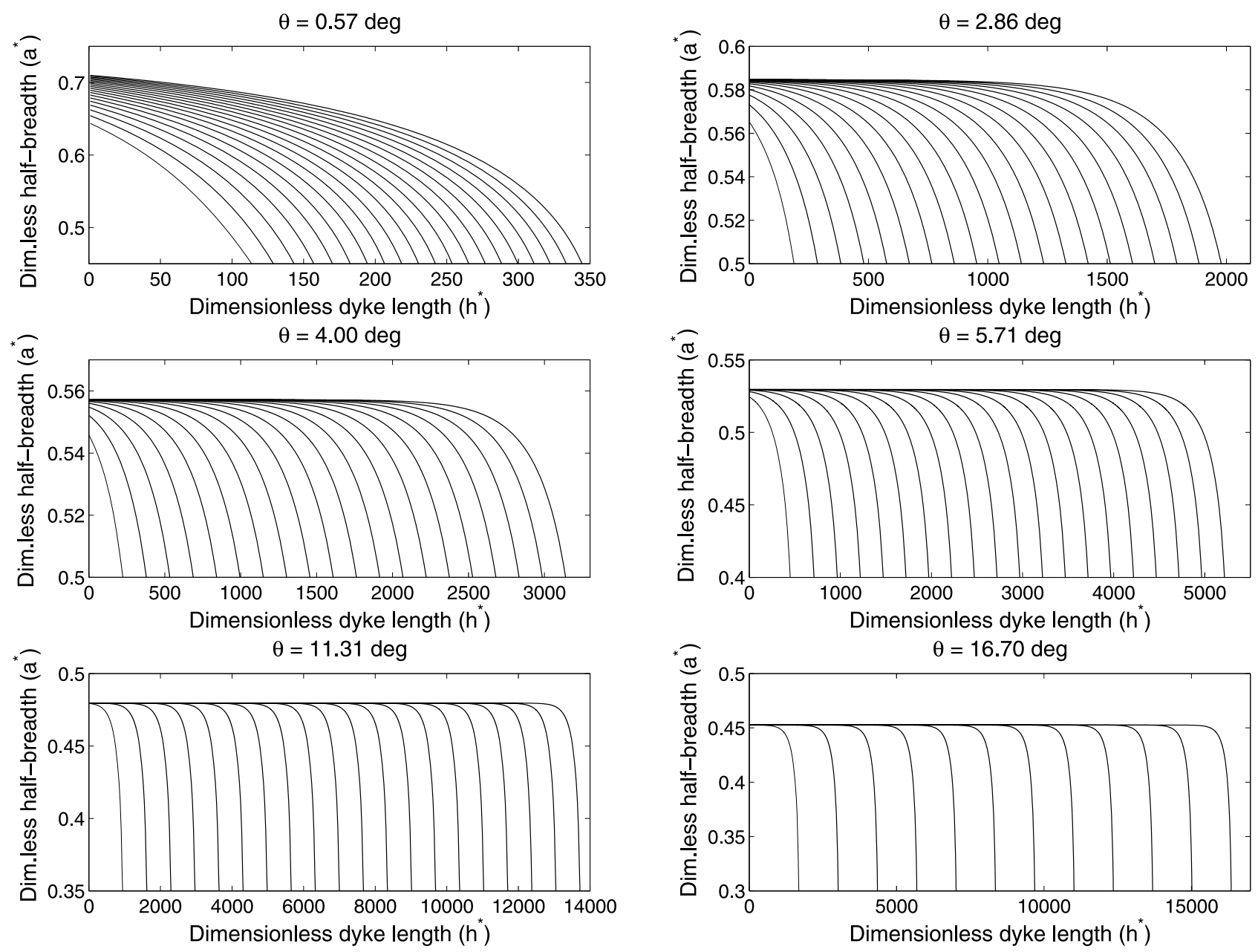

Figure 7. Lateral dike propagation: effect of the edifice flank slope on the fracture shape evolution over time. Parameters used in the calculations are $\rho_{r l}=2700 \mathrm{~kg} \mathrm{~m}^{-3}, \rho_{r u}=2300 \mathrm{~kg} \mathrm{~m}^{-3}, \rho_{m}=2400 \mathrm{~kg} \mathrm{~m}^{-3}$. Dimensionless time step between following curves is $10^{-6}$. Dimensionless numbers values are $N_{1}=$ $1.65 \times 10^{-4}$ and $N_{2}=-1.48 \times 10^{8} . N_{1}=\left(3 Q_{i n}^{3 / 4} \mu^{3 / 4} G^{9 / 4}\right) /\left(H_{b}^{9 / 4}(1-\nu)^{9 / 4}[P]^{3}\right), N_{2}=-\left(2 H_{b}^{3}(1-\nu)^{3}[P]^{4}\right) /$ $\left(3 \mu Q_{i n} G^{3}\right)$.

sense, the effect of the volcano flank slope $\theta$ is such that it carries back to the previously discussed vertical propagation case, where the breadth $2 a$ of the dike was assumed to be constant during propagation.

\section{Case Study: The 22 August 2003 Piton de la Fournaise Eruption}

\subsection{Overview on PDLF Storage and Eruptive System}

[39] The Piton de la Fournaise (PDLF), Reunion Island, Indian Ocean, is a well-studied basaltic intraplate stratovolcano, with a supply of magma from hot spots in the mantle [see, e.g., Lénat and Bachèlery, 1990; Aki and Ferrazzini, 2000; Battaglia et al., 2005; Peltier et al., 2005]. There are five conceptual models describing the shallow storage system at PDLF volcano.

[40] First, Lénat and Bachèlery [1990] propose a model of summit reservoir composed by many small independent shallow magma pockets, located above sea level at a depth of about $0.5-1.5 \mathrm{~km}$ beneath Dolomieu crater. This model is supported by the cellular automaton model of Lahaie and Grasso [1998] during the 1920-1992 period, which considers basaltic volcanoes as complex network of interacting entities at a critical state. A $1-10 \times 10^{6} \mathrm{~m}^{3}$ volume has been estimated for such magma batches through spatial extent of seismicity [Sapin et al., 1996]. This range spans the volumes of lava emitted by the eruptions occurred at PDLF in the period 1972-1992 [Sapin et al., 1996; Peltier et al., 2009], while about $32 \%$ of eruptions occurred since 1998 emitted lava volumes larger than $10 \times 10^{6} \mathrm{~m}^{3}[$ Peltier et al., 2009].

[41] Second, on crystallization arguments, Sapin et al. [1996], point out, however, that in order to produce eruptions with lava volumes of order $1-10 \times 10^{6} \mathrm{~m}^{3}$, the volume of magma in the chamber needs to be larger than the emitted volume. They therefore suggest, as a better candidate for the Piton de la Fournaise magma reservoir, the low seismic velocity zone identified by Nercessian et al. [1996] at about sea level. This aseismic zone is located just below the depth at which preeruptive seismic swarms are generally located, and extends at depths of $1.5-2 \mathrm{~km}$ below sea level. It implies a second magma chamber model volume of $1.7-4.1 \mathrm{~km}^{3}$.

[42] Third, by applying Fourier analysis of the $\mathrm{Ce} / \mathrm{Yb}$ fluctuations in the Piton de la Fournaise lavas over the 


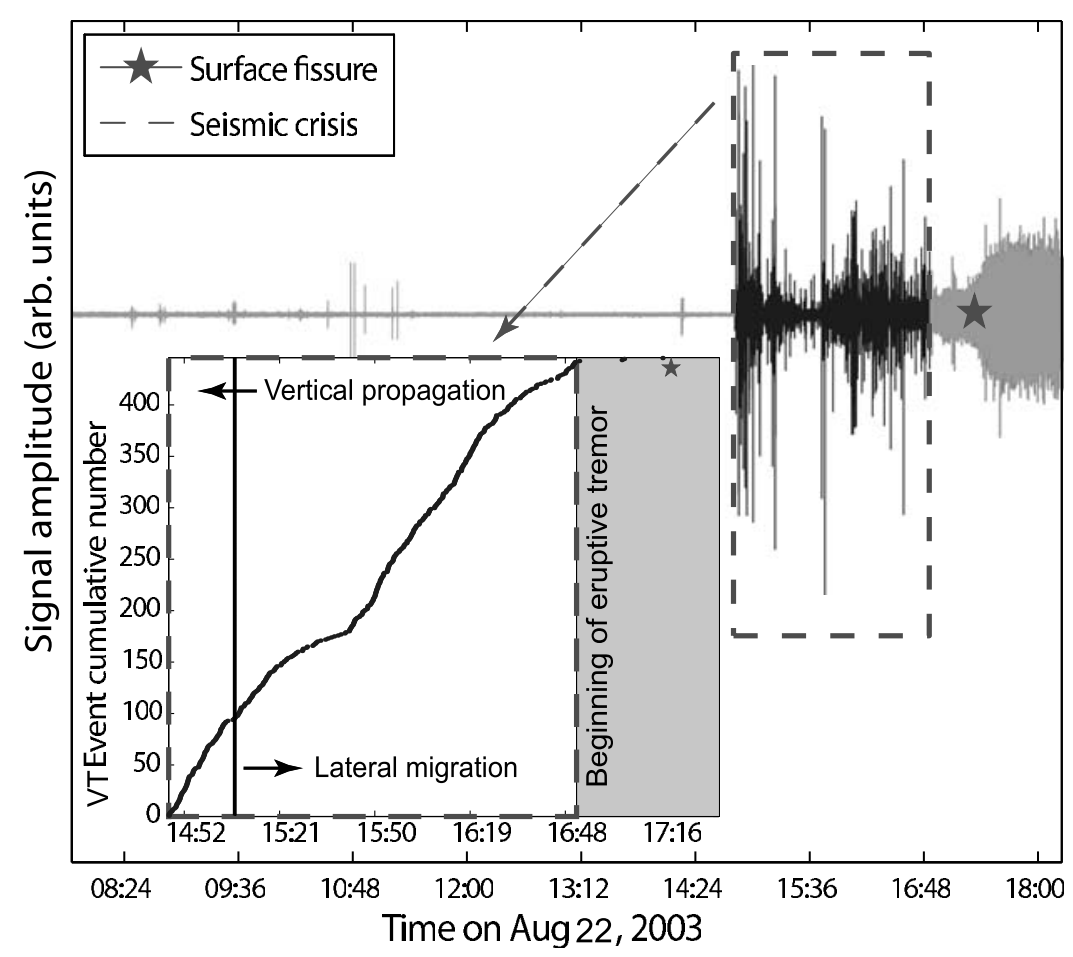

Figure 8. Seismic signal and cumulated seismicity (inset) hand-picked from continuous recordings recorded at the BOR summit station during the 22 August 2003 dike intrusion at Piton de la Fournaise volcano. Times related to the different stages of activity are from Peltier et al. [2007].

1931-1986 period, Albarède [1993] estimates a magma residence time in the reservoir between 10 and 30 years. This result, combined with magma production rates, leads Albarède to conclude that the maximum size of the PDLF magma chamber may hardly exceed $1 \mathrm{~km}^{3}$.

[43] Fourth, Sigmarsson et al. [2005] use ${ }^{238} \mathrm{U}$-series disequilibria of basalts erupted at PDLF during the period $1960-1998$ to estimate magma residence time and to infer a volume of $0.35 \mathrm{~km}^{3}$ for the Piton de la Fournaise shallow magma reservoir.

[44] Five, on tilt, extensometer and GPS data basis, Peltier et al. [2007, 2008] describe the PDLF eruptions since 2003, as fed from a common magma chamber located at a depth of 2250-2350 m beneath the summit and with a radius of $\sim 500 \mathrm{~m}$. This corresponds to a reservoir volume of about $0.5 \mathrm{~km}^{3}$. The eventuality of deeper storage systems has been discussed by Aki and Ferrazzini [2000], Battaglia et al. [2005], Prôno et al. [2009], and Peltier et al. [2009]. Hence, the presence, location, and size of reservoirs below Piton de la Fournaise still remain an open question.

[45] As discussed in previous studies [e.g., Toutain et al., 1992; Bachèlery et al., 1998; Peltier et al., 2005], flank eruptions at Piton de la Fournaise generally consist of two phases: an initial vertical rise of magma followed by a near-surface lateral migration toward the eruption site.

[46] For the 2000-2003 period, Peltier et al. [2005] observe a correlation between the duration of the lateral propagation stage and the distance of the eruptive vents from the summit. Since the seismic crisis onset coincides with the beginning of the first propagation phase [e.g., Peltier et al., 2005, 2007; Aki and Ferrazzini, 2000], Peltier et al. [2005] calculate a mean vertical speed of about $2 \mathrm{~m} \mathrm{~s}^{-1}$, while lateral migration velocities range between 0.2 and $0.8 \mathrm{~m} \mathrm{~s}^{-1}$. This results are similar to those reported by Toutain et al. [1992] for the April 1990 PDLF eruption (i.e., $2.3 \mathrm{~m} \mathrm{~s}^{-1}$ for the vertical propagation and $0.21 \mathrm{~m} \mathrm{~s}^{-1}$ for the lateral migration) and [Bachelery et al., 1998] for the eruptions taking place during the first 16 years of the PDLF Observatory (1980-1996).

[47] In this paper we focus on the August 2003 dike intrusion, which has been extensively studied through extensometer, tiltmeter, GPS and interferometric synthetic radar (InSAR) data by Peltier et al. [2005, 2007], Froger et al. [2004] and Tinard [2007]. The dike intrusion is accompanied by a seismic crisis of around 400 volcano-tectonic (VT) events within 152 min (Figure 8).

[48] Seismic data illustrated in Figure 8 confirm for the August 2003 case the seismic rate stationarity observed by Traversa and Grasso [2009] for the PDLF intrusions in the 1988-1992 period.

\subsection{Relationships Between Magma Flux Regime and Initial Conditions for Magma Reservoir}

[49] Following the results obtained in section 2.1.2 for the vertical propagation stage, and referring to the parameters listed in Table 1, we can calculate an upper bound for the reservoir initial overpressure and a lower bound for the magma reservoir volume values, such that the reservoir is able to sustain a constant influx magmatic intrusion.

[50] The upper bound for the reservoir overpressure able to sustain a constant magma flux injection, can be computed by referring to the vertical propagation stage within a homogeneous medium (i.e., we neglect the effect of the upper layer, dimensionless number $R_{2}=0$ ). We choose a large magma reservoir volume with fully compressible magma (i.e., $R_{3} \rightarrow 0$, $R_{4} \rightarrow 0$ ). The upper limit for the initial reservoir overpressure 
Table 1. Parameters Used in the Calculations for the Case of a Dike Rising in a Homogenous Medium From a Large and Fully Compressible Magma Reservoir

\begin{tabular}{lcc}
\hline \multicolumn{1}{c}{ Parameter } & Symbol & Value \\
\hline Depth of the reservoir $^{\mathrm{a}}(\mathrm{m})$ & $H$ & 2250 \\
Poisson's ratio $^{\mathrm{b}}$ & $\nu$ & 0.25 \\
Shear modulus $^{\mathrm{b}}(\mathrm{Pa})$ & $G$ & $1.125 \times 10^{9}$ \\
Rock density $^{\mathrm{b}}\left(\mathrm{kg} \mathrm{m}^{-3}\right)$ & $\rho_{r}$ & 2750 \\
Magma density $^{\mathrm{b}}\left(\mathrm{kg} \mathrm{m}^{-3}\right)$ & $\rho_{m}$ & 2400 \\
\hline
\end{tabular}

${ }^{\mathrm{a}}$ From Peltier et al. [2007].

${ }^{\mathrm{b}}$ Assumed parameters, as generic basalt values.

is given by the dimensionless number $R_{1}$ corresponding to $<5 \%$ variation in the magma flux during dike growth (see Figure 3, open squares). This is: $R_{1}<-3.55$.

[51] For parameters listed in Table 1, this implies an initial reservoir overpressure $\Delta P_{0}<2.2 \mathrm{MPa}$. Such upper limit is compatible with the average overpressure at the dike inlet estimated for the August 2003 PDLF dike intrusion, i.e., 1.7 $\mathrm{MPa}$ using InSAR data [Tinard, 2007] and at 1.1 $\mathrm{MPa}$ using GPS and tiltmeter data [Peltier et al., 2007]. Dike inlet overpressure values computed using GPS data for PDLF eruptions between 2004 and 2006 also are in the range 1.1$2.2 \mathrm{MPa}$ [Peltier et al., 2008].

[52] Note that this value is one order smaller than commonly observed rock resistances. It may be characteristic of PDLF volcano, which endured 25 eruptions in the period 1998-2007 [Peltier et al., 2009].

[53] As regarding to the generic lower bound for the magma reservoir volume able to sustain a constant magma influx intrusion, we already discussed in section 2.1.2 the influence of the dimensionless numbers $R_{3}$ and $R_{4}$ on the flux regime of the propagating dike. As shown in Figure 9 for the vertical dike propagation within a homogeneous medium case, a magma compressibility $K$ of about $1 \mathrm{GPa}$ implies that the minimum reservoir volume required for the flux of magma to remain constant over time is larger than $1 \mathrm{~km}^{3}$. The volume of magma mobilized by the lateral injection has the effect of increasing the minimum size of the magma reservoir required in order to keep the flux constant over the two-phase dike propagation. In addition, the smaller the magma chamber volume, the smaller the $R_{1}$ value necessary to keep the magma flux constant over time. For given reservoir depth, magma and rock densities, this implies smaller initial overpressures sustaining a constant influx of magma over time will be.

\subsection{Relationship Between Magma Volumes and Reservoir Overpressure Conditions}

[54] Traversa and Grasso [2009] assimilate the intrusion process on basaltic volcanoes to a strain-driven, variableloading process, reminiscent of secondary brittle creep. In such a strain-driven process, the loading is free to vary over time. It means that the overpressure at the dike inlet is free to vary over time.

[55] Most of PDLF eruptions occurring in the last decades, however, are flank eruptions, with eruptive vents located close or within the central cone [Peltier et al., 2005, 2007, 2008]. According to the model proposed by Peltier et al. [2008] for the magma accumulations and transfers at PDLF since 2000 , there is a hierarchy between the so-called

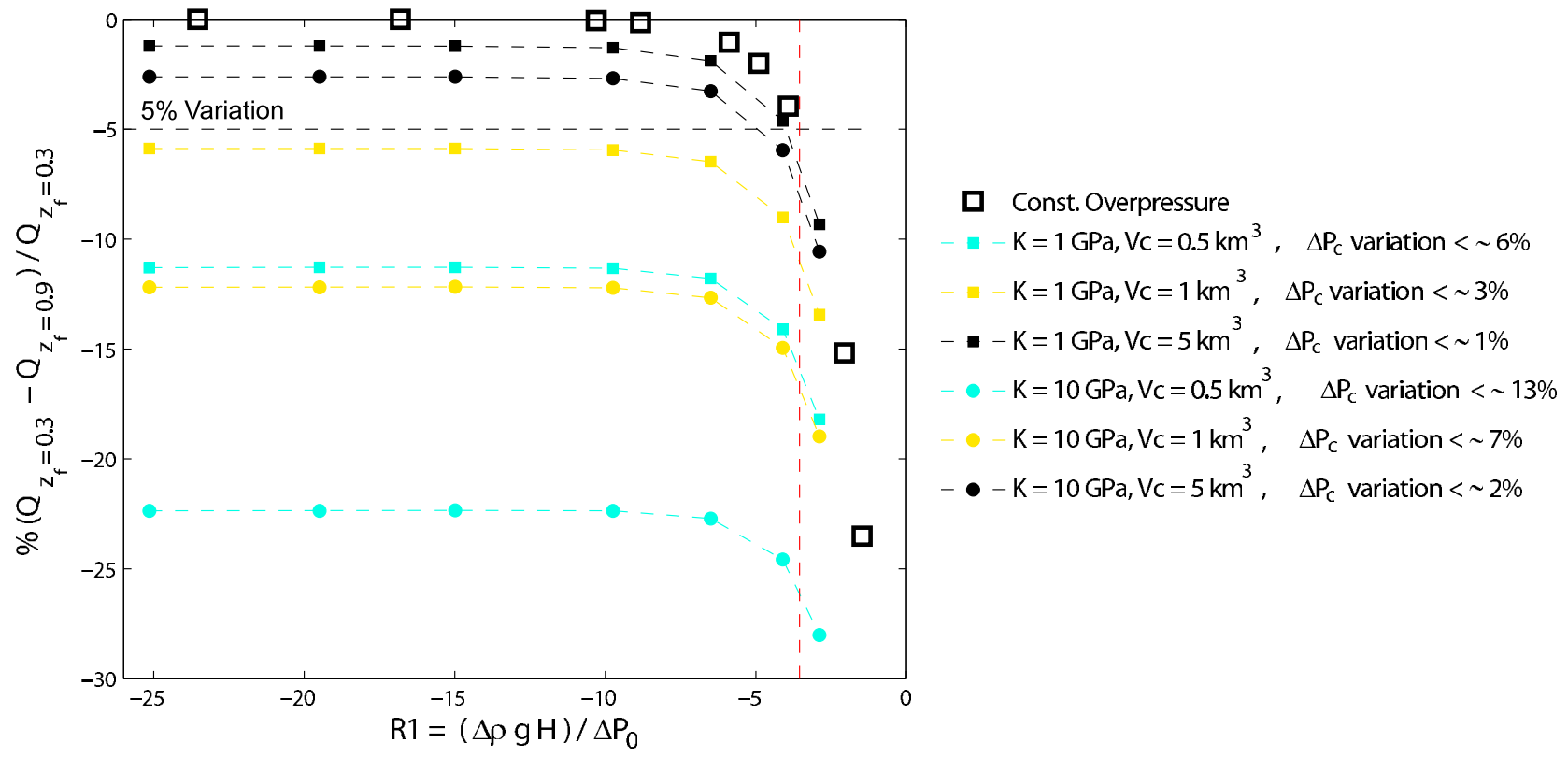

Figure 9. Interrelationship between magma influx and reservoir characteristics. Percentage of magma influx variation during dike growth within a homogeneous medium as function of the dimensionless number $R_{1}\left(R_{1}=\left(\rho_{m}-\rho_{r}\right) g H / \Delta P_{0}\right)$. Black squares indicate constant overpressure at the dike inlet; colored symbols indicate variable overpressure in the chamber. Colors are related to the $V_{c}$ value; circles or squares depend on the $K$ value. Reservoir overpressure variations $\Delta P_{c}$ variation indicated in the legend are issued from the computation. Parameter values used are $G=1.125 \times 10^{9} \mathrm{~Pa}, \nu=0.25, a=100 \mathrm{~m}, g=9.81 \mathrm{~m} \mathrm{~s}^{-2}$. $V_{c}$ values derive from conceptual models of PDLF storage system [Nercessian et al., 1996; Sapin et al., 1996; Peltier et al., 2007, 2008]. 


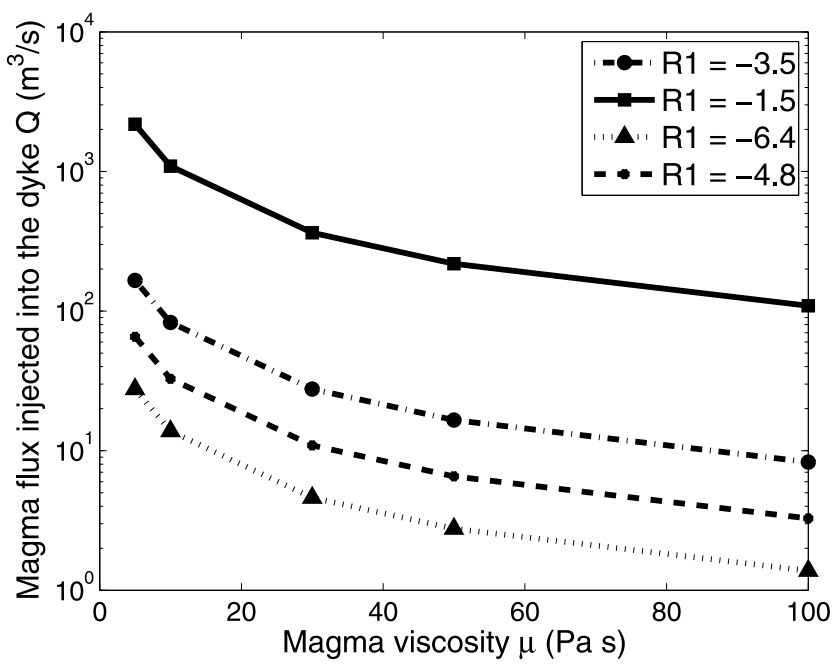

Figure 10. Dike rising vertically within a homogeneous medium from a constant overpressure magma reservoir. Magma flux injected into the dike as function of the magma viscosity and of the dimensionless number $R_{1}\left(R_{1}=\left(\rho_{m}-\right.\right.$ $\left.\left.\rho_{r}\right) g H / \Delta P_{0}\right)$. Parameters used are $H=2250 \mathrm{~m}, \rho_{m}=$ $2400 \mathrm{~kg} \mathrm{~m}^{-3}, \rho_{r}=2750 \mathrm{~kg} \mathrm{~m}^{-3}, a=100 \mathrm{~m}, \nu=0.25, G=$ $1.125 \times 10^{9} \mathrm{~Pa}$.

"distal" eruptions (occurring far from the summit cone), which release the reservoir overpressure, and 'proximal' or 'summit' eruptions (occurring close to or within the summit cone), which have negligible effect on the reservoir overpressure state. In this sense, we therefore expect most of PDLF recent eruptions to be accompanied by small variations of the magma reservoir overpressure.

[56] For the August 2003 PDLF eruption, the total amount of magma withdrawn from the reservoir (i.e., the volume of lava emitted plus the volume of the dike that keeps stuck at depth) has been estimated by Peltier et al. [2007] and Tinard [2007] at 7.2 and $7.8 \times 10^{6} \mathrm{~m}^{3}$, respectively.

[57] The model of small independent magma pockets proposed by Lénat and Bachèlery [1990] implies a substantial emptying of the lens feeding each individual eruption. This is consistent with large overpressure variations accompanying the dike intrusion. On the other hand, for the other four conceptual models proposed for the PDLF reservoir system, i.e., reservoir volumes of $1.7-4.1 \mathrm{~km}^{3}$ [Nercessian et al., 1996; Sapin et al., 1996], 0.1-0.3 km ${ }^{3}$ [Albarède, 1993], $0.35 \mathrm{~km}^{3}$ [Sigmarsson et al., 2005] and $0.5 \mathrm{~km}^{3}$ [Peltier et al., 2007, 2008], the magma volume withdrawn from the chamber during the August 2003 eruption represents between $\sim 0.2 \%$ and $\sim 2.5 \%$ of the reservoir volume. These values argue for very small overpressure variations accompanying the dike intrusion.

[58] In order to test which of these configurations (i.e., large or small overpressure variations) applies to the PDLF case, we calculate the minimum reservoir size that would be required for the overpressure to vary of a defined small percentage during dike injection. By integrating equation (15) we obtain

$$
V_{c}=\frac{\Delta V_{c}}{\exp \left(\Delta P_{c v a r}\left(\frac{4 G+3 K}{4 G K}\right)\right)-1},
$$

where $\Delta V_{c}$ is the variation in reservoir volume, $\Delta P_{c \text { var }}$ is the variation in reservoir overpressure induced by the dike intrusion, $G$ is the rock shear modulus, and $K$ is the magma bulk modulus.

[59] We assume that the volume variation induced in the magma reservoir from the August 2003 dike growth corresponds to the estimations of the dike volume, i.e., $\Delta V_{c}=1-$ $1.6 \times 10^{6} \mathrm{~m}^{3}$ [Peltier et al., 2007; Tinard, 2007]. This is related to the fact that observations of seismicity rate during dike injection [Traversa and Grasso, 2009] do not give any information about the flux evolution after the eruptive activity begins. We thus limit the validity of the constant influx model only to the dike injection, allowing that possible larger pressure and flux variations could occur during lava flow at surface. The estimated volume of lava erupted during the August 2003 eruption is $6.2 \times 10^{6} \mathrm{~m}^{3}$ [Peltier et al., 2007]. The total volume of magma withdrawn from the chamber is therefore as large as $7.2-7.8 \times 10^{6} \mathrm{~m}^{3}$.

[60] We take as the initial reservoir overpressure the upper bound we calculated previously, i.e., $\Delta P_{0}=2.2 \mathrm{MPa}$ and we compute the reservoir volume required for the magma overpressure variation $\Delta P_{c}$ variation to be the $5 \%$ of the initial reservoir overpressure, i.e., $\sim 0.085 \mathrm{MPa}$. Equation (38) gives $V_{c}=5-8 \mathrm{~km}^{3}$ as the corresponding reservoir size.

[61] When applying our model for vertical dike propagation, computations of overpressure variations induced in a realistic reservoir $\left(V_{c}=0.5-5 \mathrm{~km}^{3}\right.$ [Nercessian et al., 1996; Sapin et al., 1996; Peltier et al., 2007, 2008]) by a vertical dike fed at constant flux, are showed in Figure 3 legend. These variations are $<6 \%$, for reservoir volumes between 0.5 and $5 \mathrm{~km}^{3}$ and magma compressibility between 1 and $10 \mathrm{GPa}$.

\subsection{Relationships Between Constant Magma Influx and Dike Injection Dynamics}

[62] In this section we derive the implications of the twophase model on dike injection dynamics and we test the model for the dike intrusion that fed the August 2003 Piton de la Fournaise eruption.

[63] The August 2003 PDLF eruption involves three eruptive fissures, the first within the summit zone (at 1720 UTM), the second on the northern flank, at $2475 \mathrm{~m}$ above sea level (asl) (at 1810 UTM), and the third lower on the northern flank, at about $2150 \mathrm{~m}$ asl (at 1930 UTM) (T. Staudacher, OVPF report, 2003). The eruptive activity of the first two fissures was negligible compared to the last one (the former stopped at the end of the first day of the eruption, while only the third fissure remained active throughout the eruption) [Peltier et al., 2007; T. Staudacher, OVPF report, 2003]. As modeled by deformation data, the intrusion preceding this PDLF eruption includes a $\sim 20 \mathrm{~min}$ duration (from 1455 to 1515 UTM) vertical dike propagation followed by a $\sim 125 \mathrm{~min}$ (from 1515 to 1720 UTM) lateral injection toward the north [Peltier et al., 2007]. Although the 1720 UTM time corresponds to the opening of the first summit fracture (T. Staudacher, OVPF report, 2003), tilt data clearly indicate that the lateral dike has already fully propagated to the flank eruption site by this time. Indeed, no further evolution of the deformation is observed after 1720 UTM [Peltier et al., 2007].

[64] By inverting deformation data, Peltier et al. [2007] estimate the origin of the August 2003 dike at $400 \pm 100 \mathrm{~m}$ 


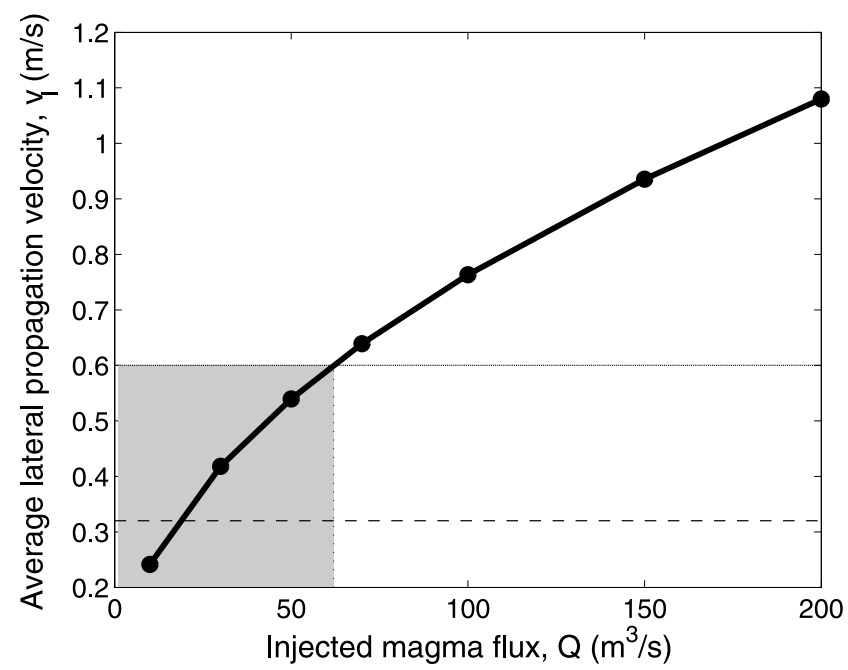

Figure 11. Lateral dike propagation: average propagation velocity versus influx of magma injected into the dike. Shaded area bounds the lateral propagation velocities estimated by Peltier et al. [2007] at Piton de la Fournaise. Parameters used are $\theta=11.8^{\circ}, \rho_{r l}=2750 \mathrm{~kg} \mathrm{~m}^{-3}, \rho_{r u}=2300 \mathrm{~kg} \mathrm{~m}^{-3}$, $\rho_{m}=2400 \mathrm{~kg} \mathrm{~m}^{-3}, H_{b}=1150 \mathrm{~m}, G=1.125 \times 10^{9} \mathrm{~Pa}$. Each magma flux value corresponds to a viscosity value, according to equation (40), where $A=4.3936$ (from the vertical homogeneous case $\left.R_{1}=-3.55\right) . R_{1}=\left(\rho_{m}-\rho_{r}\right) g H / \Delta P_{0}$.

asl, and the origin point of the lateral dike at $1500 \pm 350 \mathrm{~m}$ asl. The lateral dike travels $2.4 \pm 0.1 \mathrm{~km}$ before breaching the surface [Peltier et al., 2007]. On deformation data basis, Peltier et al. [2007] estimate an average velocity of $1.3 \mathrm{~m} \mathrm{~s}^{-1}$ for the vertical rising stage, and of $0.2-0.6 \mathrm{~m} \mathrm{~s}^{-1}$ for the lateral injection phase. The uncertainties related to vertical and horizontal propagation velocities, obtained from deformation data inversion, are $0.26 \mathrm{~m} \mathrm{~s}^{-1}$ and $0.13 \mathrm{~m} \mathrm{~s}^{-1}$, respectively (uncertainties from A. Peltier (personal communication, 2009)).

[65] In the following we calibrate the input parameters for the two-stage dike propagation model. First we derive the relationships among the parameters at stake for the two steps. Second we obtain calibrations of the same parameters by using independent estimates of dike propagation velocities in the two phases.

[66] We consider a dike rising vertically within a homogeneous medium (i.e., $R_{2}=0$ ), from a large magma reservoir with fully compressible magma (i.e., $R_{3} \rightarrow 0, R_{4} \rightarrow 0$ ). Reservoir depth $H$, magma and rock densities $\rho_{m}, \rho_{r}$ are listed in Table 1. In this case, the flux of magma injected into the dike only depends on the initial overpressure at the dike inlet and is inversely proportional to the magma viscosity, as shown in Figure 10:

$$
Q \propto \frac{1}{\mu} .
$$

When we fix the vertical velocity and we let the dike half breadth $a$ free to vary, however, we can write

$$
Q=A \mu,
$$

where

$$
A=\frac{v_{v}^{2} Q^{*} 16 H G}{v_{v}^{* 2} \Delta P_{0}^{2}(1-\nu)},
$$

$v_{v}$ is the vertical propagation velocity, $Q^{*}$ is the dimensionless flux of magma entering into the dike (i.e., $Q /[Q]$ ), and $v_{v}^{*}$ is the dimensionless vertical propagation velocity (i.e., $\left.v_{v} /[v]\right)$. The vertical propagation velocity, in turn, is given by

$$
v_{v}=C \frac{a^{2}}{\mu},
$$

where

$$
C=\frac{v_{v}^{*}(1-\nu)^{2} \Delta P_{0}^{3}}{16 H G^{2}}
$$

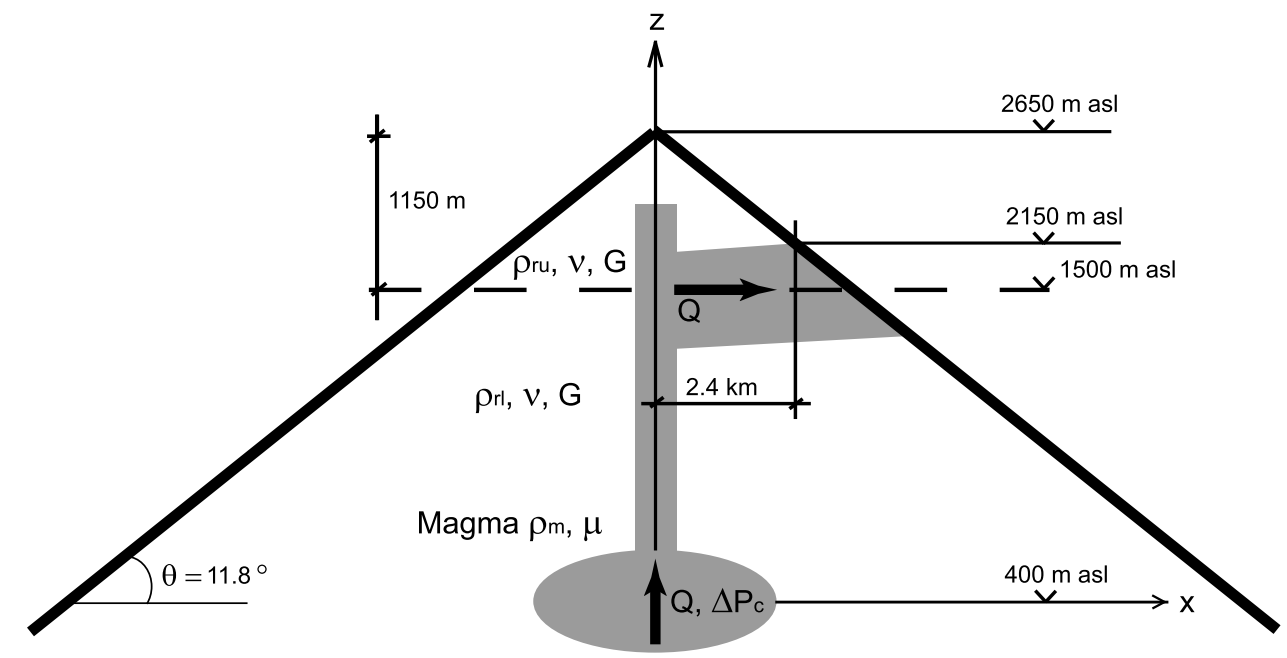

Figure 12. August 2003 PDLF case study. Sketch illustrating the geometry used in the model. Dashed line indicates input lithological discontinuity, position from Peltier et al. [2007]. Gray zones indicate magma path. All elevation data come from Peltier et al. [2007]. 
Table 2. Parameters Used in the Calculations Applied to the August 2003 Eruption at Piton de la Fournaise

\begin{tabular}{|c|c|c|}
\hline & Symbol & Value \\
\hline \multicolumn{3}{|l|}{ Parameter } \\
\hline Depth of the reservoir ${ }^{\mathrm{a}}(\mathrm{m})$ & $H$ & 2250 \\
\hline Half length of the fracture ${ }^{\mathrm{c}}(\mathrm{m})$ & $a$ & 100 \\
\hline Poisson's ratio ${ }^{\mathrm{b}}$ & $v$ & 0.25 \\
\hline Shear modulus ${ }^{\mathrm{b}}(\mathrm{Pa})$ & $G$ & $1.125 \times 10^{9}$ \\
\hline Rock density in the upper layer ${ }^{\mathrm{b}}\left(\mathrm{kg} \mathrm{m}^{-3}\right)$ & $\rho_{r u}$ & 2300 \\
\hline Rock density in the lower layer ${ }^{\mathrm{b}}\left(\mathrm{kg} \mathrm{m}^{-3}\right)$ & $\rho_{r l}$ & 2750 \\
\hline Depth of the lithological discontinuity ${ }^{\mathrm{a}}(\mathrm{m})$ & $H_{b}$ & 1150 \\
\hline Density of magma ${ }^{\mathrm{b}}\left(\mathrm{kg} \mathrm{m}^{-3}\right)$ & $\rho_{m}$ & 2400 \\
\hline Magma viscosity $^{\mathrm{c}}(\mathrm{Pa} \mathrm{s})$ & $\mu$ & 11 \\
\hline Initial magma chamber overpressure ${ }^{\mathrm{c}}(\mathrm{MPa})$ & $\Delta P_{0}$ & 1.7 \\
\hline Edifice slope $^{c}(\mathrm{deg})$ & $\theta$ & 11.8 \\
\hline Magma chamber volume ${ }^{\mathrm{d}}\left(\mathrm{km}^{3}\right)$ & $V_{c}$ & 1.7 \\
\hline Magma bulk modulus $^{\mathrm{b}}(\mathrm{Pa})$ & $K$ & $1 \times 10^{9}$ \\
\hline \multicolumn{3}{|l|}{ Dimensionless numbers } \\
\hline$R_{1 l}=\left(\rho_{m}-\rho_{r l}\right) g H / \Delta P_{0}$ & $R_{1 l}$ & -4.54 \\
\hline$R_{1 u}=\left(\rho_{m}-\rho_{r u}\right) g H / \Delta P_{0}$ & $R_{1 u}$ & 1.30 \\
\hline$R_{2}=H_{b} / H$ & $R_{2}$ & 0.51 \\
\hline$R_{3}=\left(\Delta P_{0} a^{2}(1-\nu) H\right)\left(G V_{c}\right)$ & $R_{3}$ & $1.5 \times 10^{-5}$ \\
\hline$R_{4}=4 K G /\left(\Delta P_{0}(4 G+3 K)\right)$ & $R_{4}$ & 352.90 \\
\hline
\end{tabular}

${ }^{\text {a }}$ Parameter values estimated by Peltier et al. [2007].

${ }^{\mathrm{b}}$ Assumed parameters as generic basalt values.

${ }^{\mathrm{c}}$ Derived parameters.

'Parameter values from literature [e.g., Lénat and Bachèlery, 1990; Nercessian et al., 1996; Sapin et al., 1996; Pinel and Jaupart, 2000, 2004; Peltier et al., 2008].

For a given dimensionless number $R_{1}$, the dimensionless flux and velocity (i.e., $Q^{*}$ and $v_{v}^{*}$ ) are fixed. Then, for given values of vertical propagation velocity, depth of the reservoir, and initial magma overpressure, we obtain the $A$ value.

[67] We take $R_{1}=-3.55$ (i.e., the upper limit for a $5 \%$ flux variation in the constant reservoir overpressure, homogeneous medium case as shown in Figure 3) and the parameters listed in Table 1.

[68] The lateral propagation velocity depends on the magma viscosity and on the amount of magma injected into the dike in the unit time. We then inject different magma flux and viscosity pairs into the lateral dike. Figure 11 shows how the magma flux injected in the dike is related to the lateral propagation velocity.

[69] In particular, a dike lateral propagation velocity between 0.2 and $0.6 \mathrm{~m} \mathrm{~s}^{-1}$ (shaded area in Figure 11), requires the magma flow rate injected into the laterally migrating dike to be less than about $60 \mathrm{~m}^{3} \mathrm{~s}^{-1}$. Through equation (40) this implies a magma viscosity $\mu=14 \mathrm{~Pa}$ s. This allows to constrain the value of the vertical dike half breadth $a=100 \mathrm{~m}$ (equation (42)).

[70] The value we estimate for viscosity is in good agreement with the values found by Villeneuve et al. [2008] for remolten basalts from the 1998 lava flow of the Piton Kapor, on the northern part of Dolomieu crater. Viscosity measurement experiments conducted at constant stress indicate (1) liquidus temperature of the 1998 sample at about $1200^{\circ} \mathrm{C}$ and (2) viscosities between 49 and $5 \mathrm{~Pa}$ s measured at temperatures between $1195^{\circ} \mathrm{C}$ (glass transition) and $1386^{\circ} \mathrm{C}$ (superliquidus), respectively.

[71] For the case of a dike propagating within a stratified medium from a finite size, compressible magma chamber, more parameters play a role in characterizing the dike propagation, i.e., magma bulk modulus $K$, magma chamber volume $V_{c}$, rock densities in the upper $\rho_{u}$ and lower $\rho_{l}$ layers and the depth of the lithological discontinuity $H_{b}$. We refer to the geometry illustrated in Figure 12, and we use the parameters listed in Table 2 in the calculations. Table 3 compares results issued from the computation with independent parameter estimates.

[72] From the computation we obtain a dike which rises vertically at an average velocity of $\sim 1.2 \mathrm{~m} \mathrm{~s}^{-1}$ up to the lithological discontinuity. Figure 13 shows the effect of the density barrier on the propagation of the vertical dike. It quantifies injected magma flux and volume and dike vertical propagation velocity over time (Figures 13a, 13b, and 13c). The shape of the vertical dike for different propagation steps is illustrated in Figure 13d. The flow of magma injected into the vertical dike over time is $\sim 35 \mathrm{~m}^{3} \mathrm{~s}^{-1}$, through a fracture of width $b \sim 30 \mathrm{~cm}$, which matches with the value found by Peltier et al. [2007] and Froger et al. [2004] and field observations of Peltier et al. [2007].

[73] The dike extends above the discontinuity, but its upward propagation is set back by the negative buoyancy [Pinel and Jaupart, 2004]. At the density step depth, magma overpressure grows as the dike head inflates. It may eventually exceed rock toughness and a new fracture may propagate laterally away. Here we set up a lateral dike, which propagates toward the northern flank. We assume all the magma flux rising through the vertical dike is injected into the lateral one. The slope of the edifice and the lack of lateral variation in stress gradients, allow for the dike half breadth $a$ to be constant during the lateral propagation (see Figure 7).

[74] The computed lateral dike breadth $2 a$ is $\sim 950 \mathrm{~m}$. The upper bound of the fracture breaches the surface at a height of about $2000 \mathrm{~m}$ asl after $2.3 \mathrm{~km}$ lateral propagation, in agreement with field observations of eruptive fracture location [Peltier et al., 2007; Tinard, 2007]. The average propagation velocity we compute for the lateral dike is $\sim 0.48 \mathrm{~m}$ $\mathrm{s}^{-1}$, in agreement with the upper limit value estimated by Peltier et al. [2007] by deformation data inversion ( 0.2 to $0.6 \mathrm{~m} \mathrm{~s}^{-1}$ ).

[75] We remind that the flux of magma injected in the vertical and lateral dikes is related to the respective initial dike breadth. From the computation we get lateral dike breadth $(a=476 \mathrm{~m})$ about five times the vertical dike one

Table 3. Model Validation on the August 2003 Piton de la Fournaise Eruption ${ }^{\text {a }}$

\begin{tabular}{lcc}
\hline \multicolumn{1}{c}{ Parameter } & Observation Estimate & Model Output \\
\hline Vertical average dike propagation velocity $\left(\mathrm{m} \mathrm{s}^{-1}\right)$ & $1.3 \pm 0.26^{\mathrm{b}}$ & 1.23 \\
Lateral average dike propagation velocity $\left(\mathrm{m} \mathrm{s}^{-1}\right)$ & $0.2-0.6 \pm 0.13^{\mathrm{b}}$ & 125 \\
Lateral phase duration $(\mathrm{min})$ & $2400 \pm 100^{\mathrm{b}}$ & 81 \\
Lateral covered distance $(\mathrm{m})$ & $1 \pm 0.23^{\mathrm{b}} \times 10^{6}$ & 2300 \\
Dike total volume $\left(\mathrm{m}^{3}\right)$ & $0.82 \times 10^{6}$ \\
\hline
\end{tabular}

${ }^{\mathrm{a}}$ Comparison between independent parameter estimations based on deformation data from Peltier et al. [2007] and computation results.

${ }^{\mathrm{b}}$ A. Peltier (personal communication, 2009). 

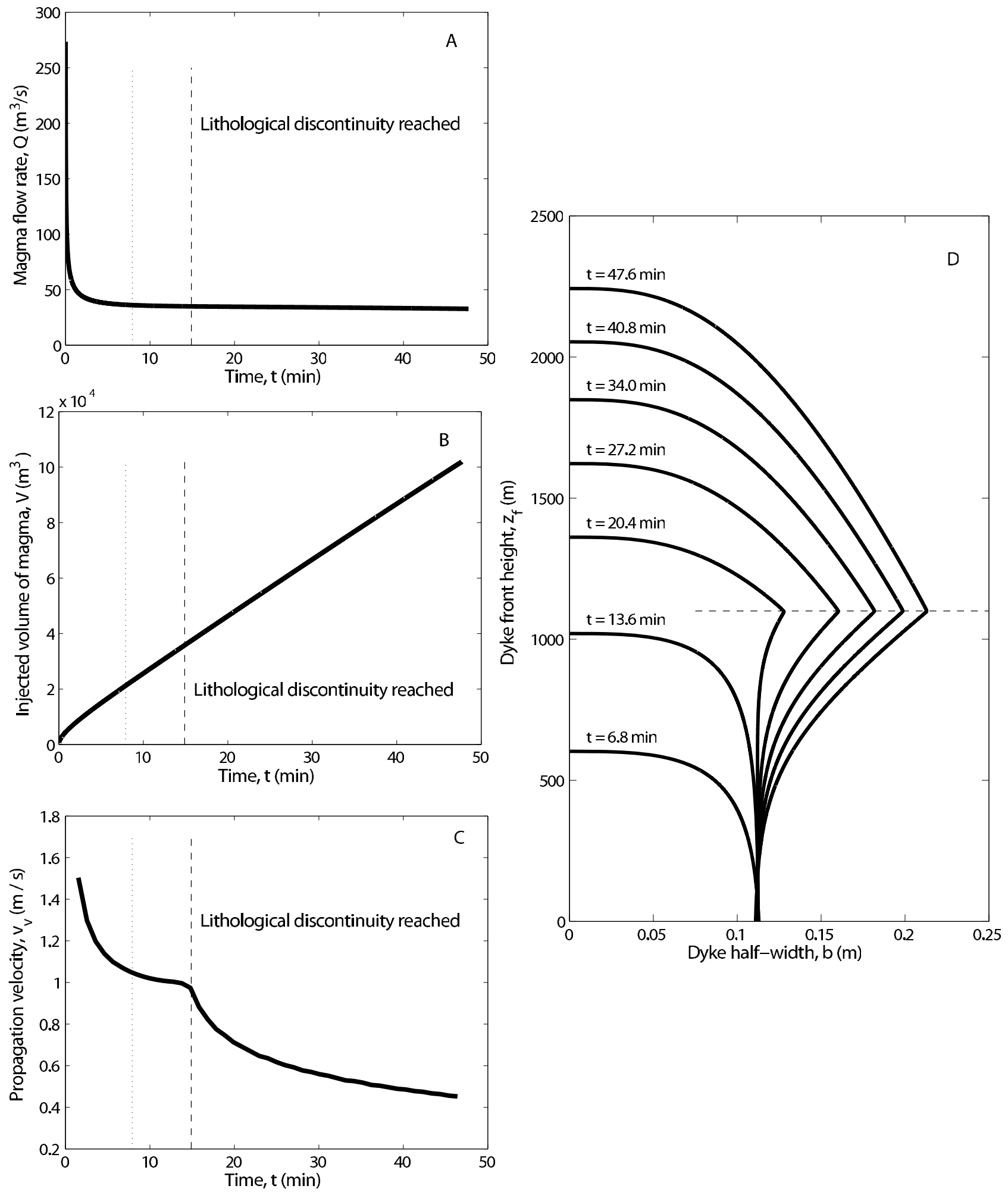

Dyke half-width, $b(\mathrm{~m})$

Figure 13. Effect of a lithological discontinuity on the vertical propagation of a magma-filled dike. (a) Magma flux injected into the dike over time; (b) dike volume (i.e., cumulative volume of magma injected into the dike over time); (c) propagation velocity versus time; (d) evolution of the crack shape for progressive growth stages. Parameter values used in the calculation are listed in Table 2. Dotted lines in Figures $13 \mathrm{a}, 13 \mathrm{~b}$, and $13 \mathrm{c}$ correspond to $z_{f} / H=z_{f}^{*}=0.3$. 
$(a=100 \mathrm{~m})$. This is related to the fact that horizontal velocity is much lower than the vertical, which has the effect of making the dike growing less along the propagation direction and to develop crosswise. The propagation velocity ratio, therefore, somehow inversely mimics the dike breadth ratio between the vertical and the lateral phases.

\section{Conclusions}

[76] Seismic observations contemporary to dike propagation on basaltic volcanoes show stationary seismicity rate during dike propagation in the last phase before an eruption, despite possible variations of the dike tip velocity [Traversa and Grasso, 2009]. Also, a clear and monotonic hypocenter migration of the seismicity contemporary to dike propagation has been rarely observed. These suggest that the observed dike-induced seismicity is the response of the edifice to the volumetric deformation induced by the magma intruding the solid matrix [Traversa and Grasso, 2009]. Accordingly, Traversa and Grasso [2009] argue for the stationary seismicity rate contemporary to the intrusion to be a proxy for a constant flux of magma entering the dike in the unit time.

[77] In order to test the implications of this assertion with respect to the volcano fluid dynamics, we implement a twophase dike propagation model, including a first vertical propagation followed by a lateral migration.

[78] We demonstrate that, although propagation velocity varies of one order of magnitude among the different propagation phases (i.e., $1.3 \mathrm{~m} \mathrm{~s}^{-1}$ and 0.2 to $0.6 \mathrm{~m} \mathrm{~s}^{-1}$ for the vertical and lateral propagation, respectively), the flow rate of magma injected into the dike can remain constant over time under given conditions. This is related both, to the fact that velocity depend on dike size for the two propagation phases, and to the evolution of dike growth, which is not limited only to elongation. It supports the idea of direct scaling between the magma flux intruding the solid and the observed seismicity rate through volumetric deformation. On the other hand it rejects a direct scaling between the seismicity rate and the dike propagation velocity. In this sense the seismicity rate recorded at low-viscosity volcanoes during dike intrusion represents the response of the solid matrix to a stationary volumetric deformation induced by the intrusion itself.

[79] Obeying the laws governing fluid dynamics, the constant magma flux can be sustained by either, a constant or a slightly variable overpressure at the base of the dike. The model we propose, however, does not allow for asserting one hypothesis with respect to the other. Indeed it allows to investigate the implications of such a stationary flux hypothesis. For the vertical propagation, once the geometry and the physical parameters are fixed, the constant influx assumption bounds the range of possible initial magma overpressures and volumes of the magma reservoir. Specifically, only a magma reservoir with sufficiently small initial overpressure and sufficiently large volume is able to sustain a dike injection fed at constant flux.

[80] The flux value computed in the vertical phase is injected in the lateral propagation phase and it determines, together with static conditions of pressure equilibrium, dike size and lateral propagation rate. In this way, the model we discuss in this paper allows to constrain the ratio between vertical and horizontal dike thickness.
[81] We validate the model in an application to the August 2003, Piton de la Fournaise eruption. It consists of two main phases: a vertical propagation, followed by a horizontal migration toward the eruption site [Lénat and Bachèlery, 1990; Toutain et al., 1992; Bachèlery et al., 1998; Bachèlery, 1999; Peltier et al., 2005, 2007, 2008]. According to the classification proposed by Peltier et al. [2008], the August 2003 PDLF eruption is a so-called "proximal" eruption, with eruptive activity concentrated on the volcano flank, close to the central cone.

[82] In this framework, the small values of initial reservoir overpressure (i.e., $\leq 2.2 \mathrm{MPa}$ ), and the small variations of this overpressure accompanying dike propagation (i.e., $\leq 6 \%$ ) we obtain from the computation, argue for this eruption to belong to an early stage of a PDLF refilling cycle [see Peltier et al., 2008]. The small overpressure variations argue for either, the volume of magma withdrawn from the reservoir during the injection to be small compared to the reservoir volume, or the magma flow rate injected into the dike in the unit time to be small compared to a possible continuous magma flow refilling the shallow reservoir from depth (as proposed by Peltier et al. [2007]).

[83] The average intrusion velocities we compute for the dikes feeding the August 2003 PDLF eruption well reproduce the values estimated by Peltier et al. [2007] on deformation data basis. It further supports the validity of our model.

[84] In conclusion, the dike propagation model we propose, allows for validating the constant magma influx initial condition as geophysically realist for volcano processes.

[85] Acknowledgments. We thank B. Taisne and A. Peltier for suggestions and interesting discussions. We acknowledge two anonymous reviewers for the care devoted to the review and the interesting and constructive remarks made. The data used in this study have been acquired by the Piton de la Fournaise Volcanological Observatory (OVPF/IPGP). Special thanks to V. Ferrazzini, in charge of the OVPF seismological network. P.T. and J.R.G. are supported by VOLUME-FP6 and TRIGS projects, contracts 08471 and 043386 , respectively.

\section{References}

Aki, K., and V. Ferrazzini (2000), Seismic monitoring and modeling of an active volcano for prediction, J. Geophys. Res., 105(B7), 16,617-16,640, doi:10.1029/2000JB900033.

Aki, K., M. Fehler, and S. Das (1977), Source mechanism of volcanic tremor: Fluid-driven crack models and their application to the 1963 Kilauea eruption, J. Volcanol. Geotherm. Res., 2, 259-287, doi:10.1016/ 0377-0273(77)90003-8.

Albarède, F. (1993), Residence time analysis of geochemical fluctuations in volcanic series, Geochim. Cosmochim. Acta, 57(3), 615-621, doi:10.1016/0016-7037(93)90372-4.

Aloisi, M., A. Bonaccorso, and S. Gambino (2006), Imaging composite dike propagation (Etna, 2002 case), J. Geophys. Res., 111, B06404, doi:10.1029/2005JB003908.

Bachèlery, P. (1999), Le Fonctionnement des volcans boucliers, Habilitation à Diriger des Recherches thesis, 698 pp., Univ. de la Reunion, Saint Denis, France.

Bachèlery, P., P. Kowalski, P. Catherine, J. Delmond, P. Blum, and J. Croce (1998), Precise temporal and mechanical identification of dyke emplacement using deformation monitoring at Piton de la Fournaise, in The European Laboratory Volcanoes: Proceedings of the 2nd Workshop, Santorini, Greece, 2 to 4 May 1996, EUR 18161 EN, edited by R. Casale et al., pp. 475-485, Eur. Comm., Brussels.

Battaglia, J., and K. Aki (2003), Location of seismic events and eruptive fissures on the Piton de la Fournaise volcano using seismic amplitudes, J. Geophys. Res., 108(B8), 2364, doi:10.1029/2002JB002193.

Battaglia, J., V. Ferrazzini, T. Staudacher, K. Aki, and J. Cheminee (2005), Pre-eruptive migration of earthquakes at the Piton de la Fournaise volcano 
(Reunion Island), Geophys. J. Int., 161(2), 549-558, doi:10.1111/j.1365246X.2005.02606.X.

Carmichael, I., J. Nicholls, F. Spera, B. Wood, and S. Nelson (1977), Hightemperature properties of silicate liquids: Applications to the equilibration and ascent of basic magma, Philos. Trans. R. Soc. London, Ser. A, 286, 373-429, doi:10.1098/rsta.1977.0124.

Cornet, F. (1992), Fracture processes induced by forced fluid percolation, in Volcanic Seismology, IAVCEI Proc. Volcanol., 3, 407-431.

Einarsson, P., and B. Brandsdottir (1980), Seismological evidence for lateral magma intrusion during the 1978 deflation of the Krafla volcano in NE Iceland, J. Geophys., 47, 160-165

Froger, J.-L., Y. Fukushima, P. Briole, T. Staudacher, T. Souriot, and N. Villeneuve (2004), The deformation field of the August 2003 eruption at Piton de la Fournaise, Reunion Island, mapped by ASAR interferometry, Geophys. Res. Lett., 31, L14601, doi:10.1029/2004GL020479.

Grasso, J., and P. Bachèlery (1995), Hierarchical organization as a diagnostic approach to volcano mechanics: Validation on Piton de la Fournaise Geophys. Res. Lett., 22(21), 2897-2900, doi:10.1029/95GL01786.

Ida, Y. (1999), Effects of the crustal stress on the growth of dikes: Conditions of intrusion and extrusion of magma, J. Geophys. Res., 104(B8), 17,897-17,909, doi:10.1029/1998JB900040.

Klein, F., R. Koyanagi, J. Nakata, and W. Tanigawa (1987), The seismicity of Kilauea's magma system, in Volcanism in Hawaii, vol. 2, U.S. Geol. Surv. Prof. Pap., 1350, 1019-1185.

Lahaie, F., and J. Grasso (1998), A fluid-rock interaction cellular automaton of volcano mechanics: Application to the Piton de la Fournaise, J. Geophys. Res., 103(B5), 9637-9649, doi:10.1029/98JB00202.

Lénat, J., and P. Bachèlery (1990), Structure et fonctionnement de la zone centrale du Piton de la Fournaise, in Le Volcanisme de La Réunion, edited by J. Lénat, pp. 257-296, Centre de Rech. Volcanol., Clermont-Ferrand, France.

Lister, J. (1990a), Buoyancy-driven fluid fracture: the effects of material toughness and of highly viscous fluids, J. Fluid Mech., 210, 263-280, doi:10.1017/S0022112090001288

Lister, J. (1990b), Buoyancy-driven fluid fracture: Similarity solutions for the horizontal and vertical propagation of fluid-filled cracks, J. Fluid Mech., 217, 213-239, doi:10.1017/S0022112090000696.

Lister, J., and R. Kerr (1991), Fluid-mechanical models of crack propagation and their application to magma transport in dykes, J. Geophys. Res., 96(B6), 10,049-10,077, doi:10.1029/91JB00600.

Maaløe, S. (1998), Shape of ascending feeder dikes, and ascent modes of magma, J. Volcanol. Geotherm. Res., 81(3-4), 207-214, doi:10.1016/ S0377-0273(98)00008-0.

McKenzie, D. (1984), The generation and compaction of partially molten rock, J. Petrol., 25(3), 713-765.

Mériaux, C., and C. Jaupart (1998), Dike propagation through an elastic plate, J. Geophys. Res., 103(B8), 18,295-18,314, doi:10.1029/ 98JB00905

Mériaux, C., J. Lister, V. Lyakhovsky, and A. Agnon (1999), Dyke propagation with distributed damage of the host rock, Earth Planet. Sci. Lett., 165(2), 177-185, doi:10.1016/S0012-821X(98)00264-7.

Muskhelishvili, N. (Ed.) (1963), Some Basic Problems of the Mathematical Theory of Elasticity: Fundametal Equations Plane Theory of Elasticity, Torsion and Bending, Noordhoff, Leyden, Netherlands.

Nercessian, A., A. Hirn, J. Lépine, and M. Sapin (1996), Internal structure of Piton de la Fournaise volcano from seismic wave propagation and earthquake distribution, J. Volcanol. Geotherm. Res., 70(3-4), 123 143, doi:10.1016/0377-0273(95)00042-9.

Nishimura, T., S. Ozawa, M. Murakami, T. Sagiya, T. Tada, M. Kaidzu, and M. Ukawa (2001), Crustal deformation caused by magma migration in the northern Izu Islands, Japan, Geophys. Res. Lett., 28(19), 3745-3748, doi:10.1029/2001GL013051.

Pasteris, J. (1984), Kimberlites: Complex mantle melts, Annu. Rev. Earth Planet. Sci., 12(1), 133-153, doi:10.1146/annurevea. 12.050184.001025.

Pedersen, R., F. Sigmundsson, and P. Einarsson (2007), Controlling factors on earthquake swarms associated with magmatic intrusions; Constraints from Iceland, J. Volcanol. Geotherm. Res., 162(1-2), 73-80, doi:10.1016/j.jvolgeores.2006.12.010

Peltier, A., V. Ferrazzini, T. Staudacher, and P. Bachèlery (2005), Imaging the dynamics of dyke propagation prior to the 2000-2003 flank eruptions at Piton de La Fournaise, Reunion Island, Geophys. Res. Lett., 32 , L22302, doi:10.1029/2005GL023720.

Peltier, A., T. Staudacher, and P. Bachèlery (2007), Constraints on magma transfers and structures involved in the 2003 activity at Piton de La Fournaise from displacement data, J. Geophys. Res., 112, B03207, doi:10.1029/2006JB004379

Peltier, A., V. Famin, P. Bachèlery, V. Cayol, Y. Fukushima, an T. Staudacher (2008), Cyclic magma storages and transfers at Piton de
La Fournaise volcano (La Réunion hotspot) inferred from deformation and geochemical data, Earth Planet. Sci. Lett., 270, 180-188, doi:10.1016/ j.eps1.2008.02.042.

Peltier, A., P. Bachèlery, and T. Staudacher (2009), Magma transport and storage at Piton de La Fournaise (La Réunion) between 1972 and 2007: A review of geophysical and geochemical data, J. Volcanol. Geotherm. Res., 184, 93-108, doi:10.1016/j.jvolgeores.2008.12.008.

Pinel, V., and C. Jaupart (2000), The effect of edifice load on magma ascent beneath a volcano, Philos. Trans. R. Soc. London, Ser. A, 358, 1515-1532, doi:10.1098/rsta.2000.0601

Pinel, V., and C. Jaupart (2004), Magma storage and horizontal dyke injection beneath a volcanic edifice, Earth Planet. Sci. Lett., 221(1-4), $245-$ 262, doi:10.1016/S0012-821X(04)00076-7.

Pitcher, W. (1979), The nature, ascent and emplacement of granitic magmas, J. Geol. Soc., 136(6), 627-662, doi:10.1144/gsjgs.136.6.0627.

Prôno, E., J. Battaglia, V. Monteiller, J. Got, and V. Ferrazzini (2009), P-wave velocity structure of Piton de la Fournaise volcano deduced from seismic data recorded between 1996 and 1999, J. Volcanol. Geotherm. Res. 184(1-2), 49-62, doi:10.1016/j.jvolgeores.2008.12.009.

Roper, S., and J. Lister (2005), Buoyancy-driven crack propagation from an over-pressured source, J. Fluid Mech., 536, 79-98, doi:10.1017/ S0022112005004337.

Rubin, A. (1993a), Dikes vs. diapirs in viscoelastic rock, Earth Planet. Sci. Lett., 119(4), 641-659, doi:10.1016/0012-821X(93)90069-L.

Rubin, A. (1993b), Tensile fracture of rock at high confining pressure: Implications for dyke propagation, J. Geophys. Res., 98(B9), 15,91915,935, doi:10.1029/93JB01391.

Rubin, A. (1995), Propagation of magma-filled cracks, Annu. Rev. Earth Planet. Sci., 23(1), 287-336, doi:10.1146/annurev.ea.23.050195.001443. Rubin, A., and D. Gillard (1998), Dike-induced earthquakes: Theoretical considerations, J. Geophys. Res., 103(B5), 10,017-10,030, doi:10.1029/ 97JB03514.

Rubin, A., D. Gillard, and J. Got (1998), A reinterpretation of seismicity associated with the January 1983 dike intrusion at Kilauea Volcano, Hawaii, J. Geophys. Res., 103(B5), 10,003-10,015, doi:10.1029/ 97JB03513.

Sapin, M., A. Hirn, J. Lépine, and A. Nercessian (1996), Stress, failure and fluid flow deduced from earthquakes accompanying eruptions at Piton de la Fournaise volcano, J. Volcanol. Geotherm. Res., 70(3-4), 145167, doi:10.1016/0377-0273(95)00043-7.

Shaw, H. (1980), The fracture mechanisms of magma transport from the mantle to the surface, in Physics of Magmatic Processes, edited by R. B Hargraves, pp. 201-264, Princeton Univ. Press, Princeton, N. J.

Sigmarsson, O., M. Condomines, and P. Bachèlery (2005), Magma residence time beneath the Piton de la Fournaise Volcano, Reunion Island, from U-series disequilibria, Earth Planet. Sci. Lett., 234(1-2), 223-234, doi:10.1016/j.epsl.2005.02.015.

Spera, F. (1980), Aspects of magma transport, in Physics of Magmatic Processes, edited by R. B. Hargraves, pp. 232-265, Princeton Univ. Press, Princeton, N. J.

Taisne, B., and C. Jaupart (2009), Dike propagation through layered rocks, J. Geophys. Res., 114, B09203, doi:10.1029/2008JB006228.

Tinard, P. (2007), Caractérisation et modélisation des déplacements du sol associés a l'activité volcanique du Piton de la Fournaise, ile de la Réunion, a partir de donées interférométriques. Aout 2003 - Avril 2007, Ph.D. thesis, Univ. Blaise Pascal-Clermont-Ferrand II, Clermont-Ferrand, France.

Toda, S., R. Stein, and T. Sagiya (2002), Evidence from the AD 2000 Izu islands earthquake swarm that stressing rate governs seismicity, Nature, 419(6902), 58-61, doi:10.1038/nature00997.

Toutain, J., P. Bachèlery, P. Blum, J. Cheminee, H. Delorme, L. Fontaine, P. Kowalski, and P. Taochy (1992), Real time monitoring of vertical ground deformations during eruptions at Piton de la Fournaise, Geophys. Res. Lett., 19(6), 553-556, doi:10.1029/91GL00438.

Traversa, P., and J. Grasso (2009), Brittle creep damage as the seismic signature of dyke propagations within basaltic volcanoes, Bull. Seismol. Soc. Am., 99(3), 2035-2043, doi:10.1785/0120080275.

Villeneuve, N., D. Neuville, P. Boivin, P. Bachèlery, and P. Richet (2008), Magma crystallization and viscosity: A study of molten basalts from the Piton de la Fournaise volcano (La Réunion island), Chem. Geol. 256(3-4), 242-251, doi:10.1016/j.chemgeo.2008.06.039.

J. R. Grasso and P. Traversa, LGIT, OSUG, Université Joseph Fourier, CNRS, BP 53, F-38041 Grenoble CEDEX 9, France. (paola.traversa@obs. ujf-grenoble.fr)

V. Pinel, LGIT, IRD, Université de Savoie, Campus Scientifique, CNRS, F-73376 Le Bourget du Lac CEDEX, France. 